\title{
CSHP Professional Practice Conference 2009: Poster Abstracts / Conférence sur la pratique professionnelle 2009 de la SCPH : Résumés des affiches
}

\section{Sunday, February 1, $2009 \bullet$ \\ Dimanche $1^{\text {er }}$ février 2009}

1. Comparaison du niveau d'accord à des énoncés sur l'éthique pharmaceutique entre des internes français et québécois

2. Fentanyl Patches: Analysis of International Medication Incidents

3. Development of a Sustainable Parenteral to Oral Antimicrobial Monitoring Protocol

4. Reversal of Parenteral Nutrition-Induced Cholestasis in Infants with Intestinal Failure Using Parenteral Fish Oil: a Canadian Single Centre Case Series

5. Affichage en temps réel de la production dans un satellite d'oncologie

6. Varenicline-Associated Symptoms of Bipolar Mania

7. Heparin Nomograms-Evaluation of Adherence and Effectiveness

8. Revue d'utilisation de la ticarcilline/acide clavulanique en soins intensifs pédiatriques

9. Évaluation d'un module web pour la localisation des médicaments d'urgence

10. Projet pilote de développement d'une banque de données d'image pour l'identification des médicaments en établissement de santé

11. Validation of a Computerized Program (the Glucommander Method) for Insulin Infusion Adjustments in a Cardiovascular Surgery Intensive Care Unit

12. Monte Carlo Simulations (MCSs) of Meropenem in Patients with Varying Degrees of Renal Function

\section{Monday, February 2, 2009 • \\ Lundi 2 février 2009}

1. Integration of a Community-Based Medication History (MedsCheck) into Peri-operative Medication Reconciliation

2. Evaluation of IV to PO Conversion Strategies in Surgical Areas

3. Validating a Nurses' Satisfaction Survey about Their Perceptions of a Surgical Pharmacist

4. Evaluation of an Interdisciplinary Approach to Benzodiazepine Tapering in a Geriatric Day Hospital

5. Creation of a Research Assistant Position as a University of Waterloo Co-op Pharmacy Student Experience

6. Hormone Replacement Therapy: An Analysis Focusing on Drug Claims by Female Seniors 2000 to 2007

7. Monitoring and Documentation of Outcomes from Targeted Interventions: An Interprofessional Quality Improvement Project

8. Can Pharmacy Students Effectively Partner with Pharmacists to Support Medication Reconciliation for Patients?

9. Computerized Prescriber Order Entry and Medication Reconciliation: Friends or Foes?

10. A Medication Reconciliation Tool

11. Dofetilide is Safe and Effective in Preventing Atrial Fibrillation Recurrences in Patients Refractory to Other Antiarrhythmic Agents Accepted for Catheter Ablation

12. Pregabalin Associated Falls in an Elderly Patient with Mildly Reduced Renal Function 


\section{Tuesday, February 3, 2009 • Mardi 3 février 2009}

1. Quality Improvement (QI) Program to Increase Adherence with Thromboprophylaxis Guidelines in Toronto Area Hospitals

2. Drug Dosing Recommendations for Continuous Renal Replacement Therapy in Tertiary References: A Critical Appraisal

3. Is Total Parenteral Nutrition with 4 and 5 Per Cent Amino Acid Solutions Safe?

4. The Guideline to Assess Pharmacotherapy Scale (GAPS): Preliminary Data

5. Topiramate-Induced Hemiplegia

6. Mortality in Febrile Neutropenic Patients Treated Initially with Cefazolin and Tobramycin

7. Evaluation of Outcomes in Patients who Received Activated Protein C (Drotrecogin alfa [Activated]) in an Intensive Care Unit: A Retrospective, Observational Study

8. Assessing the Clinical Usefulness of Checking Postprandial Glucose Levels when Fasting Glucose Levels are Near Normal in Patients with Type 2 Diabetes

9. Epoprostenol-Associated Thrombocytopenia Associated with Clinical Bleeding

10. Development and Implementation of a Pharmacy Mentorship Program

11. Development of a Chart Audit Process to Facilitate Pharmacists' Documentation

12. Systemic Reaction Associated with Nitrofurantoin Presenting as Leukocytoclastic Vasculitis

13. Development of an Evidence Based Handbook for Healthcare Providers on Exposure to Psychotropic Medications and Other Substances during Pregnancy and Lactation

\section{Wednesday, February 4, 2009 • Mercredi 4 février 2009}

1. Case Report: Sibutramine-Associated Unstable Angina

2. Analysis of Patient Controlled Analgesia and Oral Step-Down Therapy for Quality Improvement in Pain Management

3. Use of the CSHP 2015 Self-Assessment Survey to Determine Progress toward CSHP VISION 2015

4. Medication Administration Record Action Review

5. Visual Aids Enhance Inpatient Mental Health Education

6. Developing Strategies to Improve Medication Reconciliation on Hospital Admissions

7. Teaching Skills Rotation for Pharmacy Residents

8. Medication Reconciliation for Elective Surgical Admissions

9. Examination of the Long-Acting Beta-Agonist Formulary Policy and Inhaler Technique

10. Empiric Vancomycin Dosing Guidelines Based on Pharmacokinetics in Patients on Continuous Venovenous Hemodialysis

11. Cesium Induced Cardiac Arrest

12. Development and Evaluation of a Multi-hospital Structured Pharmacy Orientation Program with an Emphasis on Patient Safety

The texts of poster abstracts are published exactly as submitted by the authors and have not undergone any copyediting by the Canadian Journal of Hospital Pharmacy.

Le Journal canadien de la pharmacie hospitalière n’a pas soumis le texte des résumés des affiches à une révision linguistique et les publie ici tels que remis par les auteurs. 


\section{Comparaison du niveau d'accord à des énoncés sur l'éthique pharmaceutique entre des internes français et québécois}

Jean-François Bussières', Karin Scharr', Sonia Prot-Labarthe', Denis Lebel', Olivier Bourdon²

${ }^{1}$ Département de pharmacie et unité de recherche en pratique pharmaceutique, CHU Sainte-Justine, Montréal (Québec), et Faculté de pharmacie, Université de Montréal, Montréal (Québec)

${ }^{2}$ APHP hôpital Robert Debré et Université Paris Descartes, France

Justification : Léthique fait partie intégrante de la pratique pharmaceutique.

Objectifs : Comparer le niveau d'accord d'internes en pharmacie (France) et de résidents (Québec) à des énoncés portant sur l'éthique pharmaceutique.

Plan de l'étude et méthodologie : Enquête effectuée du $1^{\text {er }}$ avril au 25 mai 2008 Seize questions générales et 43 énoncés ont été développés (formation et études (5), recherche clinique (7), mise sur le marché et publicité (5), évaluation et données probantes (5), dispensation (4), soins pharmaceutiques (9), aspects économiques (6), déontologie (2)). Une échelle de Likert à quatre choix a été utilisée afin de mesurer le niveau d'accord/désaccord. Lissue principale est la différence entre le niveau d'accord des internes et des résidents. Les issues secondaires visent à comparer le niveau d'accord des internes de $1^{\text {ère }}$ année en France et des résidents de $1^{\text {ere }}$ au Québec et des internes en France de $1^{\text {ere }}$ et $2^{\text {ème }}$ année à celui des $3^{\text {ème }}$ et $4^{\text {ème }}$ année. Le test du chi-carré et le test exact de Fisher ont été utilisés.

Résultats : Les données utilisables recueillies proviennent de 50 répondants québécois et 158 répondants français. On note une différence statistiquement significative $(\mathrm{p} \leq 0,05)$ en ce qui concerne le niveau d'accord/désaccord regroupé pour 14 énoncés sur les 43 en comparant les résidents du Québec et les internes français notamment pour les énoncés portant sur les relations avec l'industrie pharmaceutique $(n=7)$, la place des données probantes pour les produits de santé naturels $(n=2)$, la déontologie $(n=2)$ et les relations avec les patients $(n=3)$. On note une différence significative pour 10 des 43 énoncés en comparant les $1^{\text {err }}$ année de France et du Québec. On ne note une différence significative que pour quatre énoncés entre les deux groupes d'internes français.

Conclusion : Cette étude montre qu'il existe une différence entre internes en pharmacie en France et résidents au Québec.

\section{Fentanyl Patches: Analysis of International Medication Incidents}

Roger Cheng, Carol Samples, David U, Sylvia Hyland, Certina Ho Institute for Safe Medication Practices Canada, Toronto, Ontario

Rationale: There has been a steady stream of reports in the medical literature of adverse events with fentanyl patches, including fatalities. A coordinated, system-based solution is needed to reduce errors and optimize use of this powerful opioid.

Description: On behalf of the International Medication Safety Network (IMSN), the Institute for Safe Medication Practices Canada (ISMP Canada) collated and analyzed fentanyl patch incident reports from the U.S., U. K. Canada and Ireland. A literature review was also conducted to identify other reports and system-oriented solutions.

Evaluation: In quantitative analysis $(n=3271), 8 \%$ of incidents were found to result in harm or death. Wrong dose, strength or quantity and dose omission accounted for almost two-thirds of all incidents and incidents resulting in harm or death. Administration and supply of the medication from a clinical area was the most common stage at which error occurred $(52 \%$ of all incidents and $68 \%$ of errors resulting in harm or death). Qualitative analysis $(n=1076)$ identified four major error categories: too little medication or administered too late $(42 \%$ of cases); too much medication or administered too soon (27\%); patient didn't need or should not have received the medication $(3 \%)$; and other $(27 \%)$. The "didn't need" category was associated with the highest proportion of cases resulting in harm or death (over 40\%), whereas the "too soon" category had the lowes $(<10 \%)$. For each category, the stage or process at which errors occurred and contributing factors were determined.

Conclusion: By combining results from four jurisdictions and the literature a more robust and richer analysis of the factors contributing to fentanyl patch incidents could be identified. This information can be used to develop and implement safety procedures at the patient, care interface, manufacturer and regulatory agency levels and at the stages of prescribing, order entry/transcription, dispensing, and administration/monitoring.

\section{Development of a Sustainable Parenteral to Oral Antimicrobial Monitoring Protocol}

Pamela Edmunds, Jennifer Ryan, Holly Glennie, Jennifer Sauerteig, Jaclyn LeBlanc

Atlantic Health Sciences Corporation, Saint John, New Brunswick

Rationale: Institutional antimicrobial use can be optimized through protocol implementation for conversion from intravenous (IV) to oral agents when indicated. Such programs have improved patient outcomes by reducing length of hospital stay and complications secondary to need for IV access while reducing health care costs.

Objective: To develop, implement, and assess a pharmacist-initiated IV to oral stepdown pilot program.

Study Design/Methods: A single-centre, two-phase, open label study design was utilized. Antimicrobial conversion practice data was obtained through chart review prior to program implementation and compared to post-intervention data. A protocol was developed for conversion of six IV antimicrobial agents (azithromycin, cefazolin, ceftriaxone, ciprofloxacin, metronidazole and moxifloxacin) to equivalent therapeutic oral dosing. Adult inpatient medication orders for short-term therapy were eligible for study inclusion, while orders for antimicrobial prophylaxis, treatment of febrile neutropenia and treatment of infections caused by multi-drug resistant organisms were excluded. Chi-squared analysis was used to identify differences between groups in demographics and in treatment indication, and primary outcome data was compared using a two-tailed t-test. An alpha level of 0.05 was determined to be statistically significant. A sample size of 60 eligible medication orders in each group was required to achieve a power of 0.90 .

Results: Pre-intervention, 77 patient charts were reviewed to identify 85 medication orders eligible for conversion. Physicians enrolled 65 patients with 82 eligible medication orders after protocol implementation. Patients received $4.51 \pm 2.35$ days of IV therapy on average before drug discontinuation or conversion to an oral agent, compared to $5.75 \pm 3.56$ days before protocol implementation $(\mathrm{p}<0.001)$. Mean total length of antibiotic therapy was unchanged. Total cost of antimicrobial treatment decreased by an average of $\$ 73.98$ per medication order $(\mathrm{p}<0.001)$.

Conclusion: Program implementation decreased IV antibiotic use and drug costs at the study site.

\section{Reversal of Parenteral Nutrition-Induced Cholestasis in Infants with Intestinal Failure Using Parenteral Fish Oil: a Canadian Single Centre Case Series}

Irina Karagodina, Denise Reniers, Paul Atkison, Suzanne Ratko London Health Sciences Centre, London, Ontario

Rationale: Parenteral nutrition (PN) is the mainstay of therapy for infants with intestinal problems precluding or limiting enteral feeding. Hepatic cholestasis occurs in $40-60 \%$ of infants requiring long term PN. Reversal of PN-associated liver disease using parenteral fish oil (PFO) has been documented in several case reports. We report here our institution's experience with PFO in infants with intestinal failure and $\mathrm{PN}$-induced cholestasis.

Description: Our series involves eight infants that started PFO therapy on day 13 of life $\left(38^{3} / 7\right.$ weeks postconceptional age) to 7.6 months old; data was collected over a 2.2 year period. All patients had developed hepatic cholestasis associated with PN and were treated with PFO emulsion in place of the usual soybean oil based preparation over a range of 4.6 to 8.4 months. PFO was initiated at $0.5 \mathrm{~g} / \mathrm{kg}$ and increased to $1 \mathrm{~g} / \mathrm{kg}$ after two days. Seven out of 8 infants were receiving part of their caloric intake in the form of enteral feeds. PFO therapy achieved reversal of PN-induced cholestasis (serum direct bilirubin level of $<3.4 \mu \mathrm{mol} / \mathrm{L}$ ) in all patients in a median of 130 days (64 to 193), while total bilirubin normalized in a median of 99 days (42 to 166). Therapy was well tolerated and not associated with infusion-related adverse events, clinical essential fatty acid deficiency or growth delay.

Evaluation of the Literature: A handful of case reports and a small cohort study have documented success of PFO therapy in reversing hepatic cholestasis. Our experience of reversal of cholestasis despite continued PN in a median of 18.6 weeks is consistent with previously published reports.

Importance to pharmacy practitioners: PFO emulsion appears to be effective in the treatment of PN-induced cholestasis, reducing the potential need for other medical interventions including liver/bowel transplantation. 


\section{Affichage en temps réel de la production dans un satellite d'oncologie}

\section{JeDenis Lebel', Jean-François Bussières}

'Département de pharmacie et unité de recherche en pratique pharmaceutique, CHU Sainte-Justine, Montréal (Québec); Faculté de pharmacie, Université de Montréal, Montréal (Québec)

Justification du rapport : La préparation de médicaments dangereux est centralisée en pharmacie d'oncologie. Le personnel soignant peut contacter par téléphone la pharmacie pour vérifier si les préparations de médicaments sont prêtes. Ces appels génèrent de nombreuses interruptions de travail.

Description du concept : En combinant des informations recueillies par un lecteur de code barre et les informations des banques de données du dossier pharmacologique informatisé (Gespharx®), nous avons développé une section de l'intranet pharmacie accessible au personnel soignant permettant l'affichage web de l'état de la production des préparations.

Mesures prises : Chaque étiquette d'identification des médicaments produite par la pharmacie contient un code-barres incluant un numéro unique de transaction correspondant au service. Ces code-barres sont lus après validation des ordonnances par le pharmacien et lors de la remise du médicament au personnel soignant. L'information est enregistrée dans une banque de donnée SQL Server. Une page web a été programmée en utilisant la technologie ASP afin de faire la correspondance entre les numéros de services et la dernière action réalisée et le dossier pharmacologique. Pour chaque ordonnance, l'affichage web précise le numéro de chambre, le descriptif de nom du médicament par ordonnance (i.e. nom générique, forme, teneur, format) et l'état (i.e. prêt et non remis, prêt et remis au personnel soignant).

Évaluation du projet : Le projet a été réalisé sans frais avec les ressources internes à partir de vues des tables du dossier pharmacologique informatisé avec l'autorisation du fournisseur de ce logiciel. Le développement (16 heures) et l'implantation (4 heures) ont été effectués en février 2008. Le personnel des unités de soins et de la pharmacie est très satisfait de ce nouvel outil.

Importance et utilité du concept : Le concept d'affichage web de la production de préparations stériles en oncologie est applicable en pharmacie hospitalière et peut être réalisé à peu de frais.

\section{Varenicline-Associated Symptoms of Bipolar Mania}

Rochelle Myers, Sally Ginson-Duke

The Moncton Hospital, South-East Health Services, Regional Health Authority $B$

Rationale for Case Report: Varenicline is a drug approved by Health Canada for smoking cessation. Post-marketing reports describe worsening of pre-existing psychiatric illnesses following varenicline exposure. We present 2 cases of bipolar illness requiring hospitalization, believed to be triggered in part by varenicline use. Description of Case: A 33 year-old female with a history of depression was brought to the emergency room (ER) by family, as she was acting bizarre and out of character. Her entrance diagnosis was bipolar I disorder, manic episode. Further questioning revealed that varenicline was taken for 4 days during the week prior to admission. A 43 year-old female with a history of mood-disorder was brought to the ER by police after threatening to commit suicide. The patient was admitted to hospital with a diagnosis of bipolar II disorder, hypomanic episode. Six days prior to admission, varenicline was initiated for 3 days and then discontinued.

Assessment of Causality: Varenicline is a partial agonist at $\alpha 4 \beta 2$-nicotinic receptors. Agonistic activity on dopaminergic neurons results in continual lowlevel dopamine release, which may correlate with the onset of mania and hypomania in these patients. A Naranjo score of 3 suggests a possible varenicline adverse drug reaction.

Evaluation of the Literature: Three published case-reports of vareniclineinduced mania or hypomania were located. Two cases had past history of bipolar disorder, and one had positive history of depression. All cases required hospitalization. Symptom onset occurred shortly following varenicline initiation, and resolved after discontinuation and pharmacologic treatment.

Importance of Case to Pharmacy Practitioners: These cases illustrate the importance of thorough and consistent screening for personal and family history of mood disturbances prior to varenicline use, and the need for diligent monitoring for mood and behaviour changes during therapy.

\section{Heparin Nomograms-Evaluation of Adherence and Effectiveness}

Christine Li, ${ }^{1}$ Patti Cornish ${ }^{2}$

'Pharmacy Student, University of Toronto, Ontario

2Pharmacist, Patient Safety Service, Sunnybrook Health Sciences Centre, Toronto, Ontario

Rationale: Numerous studies have evaluated the value of heparin nomograms in improving anticoagulation in various indications. Compared with empiric dosing of heparin, therapeutic anticoagulation has been demonstrated to be achieved faster and maintained better with the use of a nomogram. Two weight-based heparin nomograms have been in use at our institution for more than 10 yearsgeneral nomogram for use in thromobembolic disorders and a cardiology nomogram for patients with acute coronary syndrome (ACS).

Objectives: To evaluate the utility of our institutional heparin nomograms in everday practice.

Methods: Over a two-month period, we collected data on 53 patients being treated with one of the heparin nomograms (for at least 24 hours) to determine: (i) adherence to the instructions regarding dosing and dose adjustment; and (ii) rates of achievement of therapeutic anticoagulation (i.e., activated partial thromboplastin time (aPTT) in target range).

Results:

\begin{tabular}{lcc} 
& General Nomogram & ACS Nomogram \\
\hline Number of patients & 29 & 24 \\
Mean duration of heparin (days) & 4 & 5 \\
\% of doses deviating from nomogram & 13 & 15 \\
\% of aPTTs in target range & 28 & 40 \\
\% of aPTTs > target range & 31 & 40 \\
\% of aPTTs < target range & 41 & 20 \\
\hline
\end{tabular}

Conclusions: Overall, the results indicate that there is reasonably good adherence with the nomograms as $\leq 15 \%$ of initial doses or dosing adjustments deviated from the instructions. However, the achievement of consistent aPTTs in the target range was less than optimal. Further evaluation of both heparin nomograms is recommended to determine if dosing regimens can be improved to optimize rates of therapeutic anticoagulation.

\section{Revue d'utilisation de la ticarcilline/acide clavulanique en soins intensifs pédiatriques}

Sébastien Ruaud', Karine Touzin', Marc Lebel', Christopher Marquis', Catherine Litalien', Jean-François Bussières'

'Département de pharmacie et unité de recherche en pratique pharmaceutique, CHU Sainte-Justine, Montréal (Québec); Faculté de pharmacie, Université de Montréal, Montréal (Québec)

${ }^{2}$ Département de pédiatrie - service des soins intensifs pédiatriques, CHU Sainte-Justine, Montréal (Québec)

Justification : L'utilisation d'antibiotiques à large spectre doit être limitée afin d'éviter la résistance bactérienne.

Objectif : Décrire et évaluer l'usage de la combinaison ticarcilline/acide clavulanique (Timentin $\left.{ }^{\circledR}\right)$ dans une unité de soins intensifs pédiatriques (SIP).

Plan et méthodologie : Étude descriptive et rétrospective divisée en deux volets. Un premier volet décrit la population pédiatrique recevant du Timentin ${ }^{\circledR}$ et le profil pharmacologique et biologique des patients. Un second volet évalue la conformité par rapport à la règle d'utilisation en vigueur en ce qui concerne la dose et la fréquence d'administration et la présence d'une consultation avec un spécialiste du service de maladies infectieuses avant l'initiation de la thérapie. Les patients atteints de maladies oncologiques ou ayant reçu une greffe hématopoïétique ont été exclus.

Résultats : La revue inclut les 30 derniers patients ayant reçu une ordonnance de Timentin ${ }^{\circledR}$ aux SIP avant le 2008-07-19. La médiane d'âge des patients étudiés est de 10 mois [ $<1$ mois à 17 ans]. Tous les patients ont eu un bilan septique complet avant l'initiation du Timentin ${ }^{\circledR}$ et $80 \%$ étaient intubés avant le début du traitement. Parmi ces cultures, $66 \%$ se sont avérées positives $(80 \%$ sécrétions endotrachéales, $10 \%$ culture d'urine, $5 \%$ hémoculture, $5 \%$ cultures autres). Le Timentin ${ }^{\circledR}$ a été débuté en moyenne $17 \pm 45$ jours après l'admission aux SIP (médiane: 4, [0-247]). La durée médiane de traitement est de 7 jours [3-23 jours]. La conformité obtenue est de $47 \%$ pour le recours à une consultation en maladies infectieuses, $87 \%$ pour la fréquence de traitement et $90 \%$ pour la dose. Conclusion : Un pourcentage important de patients recevant du Timentin ${ }^{\circledR}$ aux SIP présente une culture positive aux niveau des sécrétions endotrachéales, suggérant la présence d'une pneumonie nosocomiale. Sachant que ce type d'infection est souvent difficile à confirmer chez la population pédiatrique, il apparaît difficile à ce stade-ci de limiter l'utilisation du Timentin ${ }^{\circledR}$ pour cette indication. 


\section{Évaluation d'un module web pour la localisation des médicaments d'urgence}

Serge Voytenko', Denis Lebel', Jean-François Bussières'

'Département de pharmacie et unité de recherche en pratique pharmaceutique, CHU Sainte-Justine, Montréal (Québec); Faculté de pharmacie, Université de Montréal, Montréal (Québec)

Justification du rapport : Le délai d'intervention de l'équipe de réanimation lor d'une urgence cardio-vasculaire en établissement est un facteur essentiel influençant la survie des patients. Ce délai est influencé par la capacité de localiser rapidement les médicaments requis.

Objectifs : L'objectif de cette étude est d'évaluer l'utilité d'un module web de visualisation du contenu des plateaux complets ( 43 items) et partiels (22 items) de médicaments d'urgence.

Plan et méthodologie : Il s'agit d'une étude descriptive et qualitative. Afin d'évaluer le module, nous avons invité 10 professionnels de chaque groupe (médecin, infirmière, médecin-résident, pharmacien, pharmacien-résident, assistant-technique en pharmacie) à identifier le plus rapidement possible une séquence de 30 puis de 15 médicaments affichés de façon aléatoire. Lissue principale est le temps moyen requis pour localiser chaque item. Les issues secondaires sont le temps moyen par médicament/professionnel et la comparaison du niveau d'accord à des énoncés qualitatifs en pré et post.

Résultats : Le temps moyen requis pour localiser un médicament varie de 6,0 \pm $2,6 \mathrm{sec}$ (atropine) à $23,7 \pm 20,4 \mathrm{sec}$ (vasopressine). Seuls 12 items nécessitent en moyenne moins de 10 secondes pour être localisés. Le temps moyen/item/professionnel en secondes pour un plateau complet varie selon le groupe étudié soi 10,2 (pharmacien-résident), 10,4 (pharmacien), 12,1 (médecin-résident), 12,7 (assistant-technique), 14,0 (médecin), 14,4 (infirmière). ( $p<0,0001)$. On note une différence d'accord en ce qui concerne les trois énoncés qualitatifs en pré et post, appuyant la démarche d'implantation du module en ligne à l'échelle de l'établissement. Lamajorité des répondants considère que le module devrait faire partie de la formation continue des groupes étudiés.

Conclusion : Il n'existe pas de données sur l'utilité d'un module web de visualisation de contenu de médicaments d'urgence. Cette étude montre que le module peut être utilisé pour monitorer et former le personnel soignant à la localisation du contenu de médicaments d'urgence.

\section{Projet pilote de développement d'une banque de données d'image pour l'identification des médicaments en établissement de santé \\ Serge Voytenko', Denis Lebel', Jean-François Bussières' \\ 'Département de pharmacie et unité de recherche en pratique pharmaceutique, CHU Sainte-Justine, Montréal (Québec); Faculté de pharmacie, Université de Montréal, Montréal (Québec)}

Justification du rapport : u Canada, il n'existe pas de banques de données exhaustives d'images des médicaments disponibles associées aux autres données pertinentes du produit comme le DIN. Cette information est primordiale pour rendre le circuit du médicament plus sécuritaire.

Description du concept : Développer un processus de gestion de l'identité numérique des médicaments. L'identité numérique est définie comme étant toute information reliée à un produit et enregistrée sous forme numérique. Cette identité peut comporter différents numéros d'identification, des images du produit ou des fichiers sonores de la prononciation de son nom. Dans le cadre de ce projet, nous avons ciblé les images de l'emballage externe et du plus petit format de distribution ainsi que l'apparence des formes orales liquides.

Mesures prises : Un laboratoire de photo a été développé afin de produire des images de qualité de chaque forme posologique. Une banque de données constituée de 277 images regroupe un total de 138 produits. Un processus de gestion de l'identité numérique a été défini de la réception d'un produit à la réserve de la pharmacie jusqu'à son administration par l'infirmière au chevet du patient. Une phase pilote a été mise en place afin d'assurer la mise à jour de la banque de données lorsqu'un nouveau produit acheté au sein de l'établissement n'est pas reconnu. Les produits inconnus sont mis en quarantaine, pour prise de photo et ajout à la banque de données.

Évaluation du projet : Le projet pilote a été réalisé en tenant compte du développement de la banque de données ( 8 heures), de la prise de photo, de la saisie dans la banque de données ( 400 heures initialement) et de l'implantation (40 heures).

Importance et utilité du concept : Ce projet pilote démontre la faisabilité d'une banque de données d'images pour l'identification de médicaments en établissement de santé.

\section{Validation of a Computerized Program (the Glucommander Method) for Insulin Infusion Adjustments in a Cardiovascular Surgery Intensive Care Unit}

Sharon Yamashita, Emily Ng, Frank Brommecker, Jay Silverberg, Ray Fung, Neill Adhikari, Rob Fowler

Departments of Pharmacy, Critical Care Medicine and Endocrinology, Sunnybrook Health Sciences Centre, Toronto, Ontario

Objectives: To evaluate the efficacy and safety of a computerized system (Glucommander method) for individualizing insulin infusion therapy in a Cardiovascular Surgery Intensive Care Unit (CVICU)

Methods: In a prospective, before-after cohort study, 50 patients receiving insulin according to the Glucommander system were compared to the control group $(n=50)$ who received insulin according to the standard CVICU insulin nomogram.

Results: There was no significant difference between the two groups with respect to time to target blood glucose $(5.1-8.0 \mathrm{mmol} / \mathrm{L})$, percentage of time within target range or mean amplitude of glucose excursion (MAGE). There was a reduction in percentage of time spent above target range $(\mathrm{P}<0.01)$ and an increase in percentage of time spent below target range $(\mathrm{P}<0.01)$ in the Glucommander group, with an overall reduction in mean glucose $(7.9$ vs $8.6 \mathrm{mmol} / \mathrm{L}, \mathrm{p}<0.01)$. There were a greater number of hypoglycemic episodes in the Glucommander group (3.7 versus $1.4 \%$; $\mathrm{p}<0.01)$. Nursing satisfaction surveys revealed that the program was well accepted by the nursing staff in the CVICU.

Conclusion: A computerized insulin nomogram is an effective, easy to use instrument for tighter glucose control in CVICU patients. The use of the Glucommander results in a lower mean blood glucose but an increase in hypoglycemic episodes in CVICU patients.

\section{Monte Carlo Simulations (MCSs) of Meropenem in Patients with Varying Degrees of Renal Function}

Sheryl Zelenitsky, ${ }^{1}$ Rosemary Zvonar, ${ }^{2}$ Rob Ariano ${ }^{3}$

'University of Manitoba, Winnipeg, Manitoba; 'The Ottawa Hospital,

Ottawa, Ontario; ${ }^{3}$ St. Boniface General Hospital, Winnipeg, Manitoba

Rationale: For $\beta$-lactams, maintaining concentrations above the minimum inhibitory concentration (or T>MIC) for at least $40 \%$ of the dosing interval is considered necessary to achieve positive clinical outcomes. The target may be higher, exceeding $80 \% \mathrm{~T}>\mathrm{MIC}$, for immunocompromised patients. Prolonging infusion time is one strategy used to improve pharmacodynamic target attainment (TA) especially for agents with short half-lives. Although prolonged infusions (PIs) of meropenem have been evaluated in patients with normal renal function, there is little information in those with renal dysfunction.

Objective: This analysis was performed to develop dosing recommendations for meropenem using PIs in patients with varying degrees of renal function.

Methods: MCSs of 5000 patients were generated using SYSTAT v.12. Meropenem doses with standard $(0.5 \mathrm{~h})$ and PIs $(3 \mathrm{~h})$ were compared for three levels of renal function: $51-100,26-50$, and $10-25 \mathrm{~mL} / \mathrm{min}$. A meropenem population pharmacokinetic model, and MIC distributions for Pseudomonas aeruginosa from North American surveillance data were used. TA was defined as achieving $>40 \%$ and $>80 \%$ T $>$ MIC.

Results: TA results stratified according to renal function are presented below: TA for $\geq 40 \%$ T $>$ MIC TA for $\geq 80 \%$ T $>$ MIC

$\mathrm{NCrCl}$ 51-100 $\mathrm{ml} / \mathrm{min}$

\begin{tabular}{|c|c|c|}
\hline $1 \mathrm{~g} \mathrm{Q} 8 \mathrm{H}^{*}$ & $99.8 \%$ & $80.9 \%$ \\
\hline 1 g Q8H PI & $100 \%$ & $93.0 \%$ \\
\hline $500 \mathrm{mg}$ Q8H PI & $99.7 \%$ & $82.6 \%$ \\
\hline $500 \mathrm{mg}$ Q6H & $99.6 \%$ & $85.6 \%$ \\
\hline \multicolumn{3}{|l|}{$\mathrm{CrCl} 26-50 \mathrm{ml} / \mathrm{min}$} \\
\hline $1 \mathrm{~g} \mathrm{Q12 \textrm {H } ^ { * }}$ & $99.8 \%$ & $81.4 \%$ \\
\hline $1 \mathrm{~g} \mathrm{Q12H} \mathrm{PI}$ & $100 \%$ & $89.6 \%$ \\
\hline $500 \mathrm{mg}$ Q12H PI & $99.1 \%$ & $77.5 \%$ \\
\hline 500 mg Q8H & $99.9 \%$ & $90.6 \%$ \\
\hline \multicolumn{3}{|l|}{$\mathrm{CrCl} 10-25 \mathrm{ml} / \mathrm{min}$} \\
\hline 500 mg Q12H* & $100 \%$ & $92.4 \%$ \\
\hline $500 \mathrm{mg}$ Q12H PI & $100 \%$ & $95.4 \%$ \\
\hline
\end{tabular}

* manufacturer's recommended dose

Conclusion: Based on this MCS study, meropenem dosing in patients with $\mathrm{CrCl}$ $51-100$ and $\mathrm{CrCl} 26-50 \mathrm{ml} / \mathrm{min}$ with $500 \mathrm{mg} \mathrm{Q} 8 \mathrm{H}$ and $500 \mathrm{mg} \mathrm{Q} 12 \mathrm{H}$, respectively, infused over 3 hours, are reasonable alternatives. For patients with $\mathrm{CrCl}$ of $10-25 \mathrm{ml} / \mathrm{min}$, TA was not enhanced with the PI. Patients with neutropenia, critical illness or less susceptible infections (e.g., MICs $\geq 1$ ) may benefit from PIs of higher doses. 


\section{Integration of a Community-Based Medication History (MedsCheck) into Peri-operative Medication Reconciliation}

Emily Charlesworth, Kieu Mach, Sandy Hicks, Kristine Kizemchuk, Valerie Leung, Carmine Stumpo

Toronto East General Hospital, Toronto, Ontario

Rationale: Medication safety along the continuum of care is dependent on the quality of medication information at each point of transfer. The purpose of ou study was to assess the impact of integrating a community-based medication history (MedsCheck) in peri-operative medication reconciliation for elective orthopedic surgery patients.

Objectives: The primary objective was to determine if medication reconciliation in the pre-admission clinic (PAC) using MedsCheck would reduce the number of post-operative unintentional medication discrepancies. Secondary objectives were to assess community pharmacist participation and patient satisfaction.

Study Design and Methods: Patients scheduled for elective hip and knee surgery between April-September 2008 were identified as the study population. Patients and their respective community pharmacies were contacted in advance to coordinate the MedsCheck review prior to the PAC visit. At the PAC visit, the MedsCheck document was used to prepare a best possible medication history which was documented in the patient chart. Medications were reconciled postoperatively. Results were compared to a baseline sample prior to implementation. Both patients and pharmacists were surveyed to elicit feedback on the process.

Results: Eighty-two patients were included in the study. The average number of medications was 8.9 per patient. The percentage of patients with at least 1 unintentional medication discrepancy decreased from $68.4 \%$ (13/19) to $47.6 \%$ $(39 / 82)$ post-intervention. Total unintentional discrepancies decreased from $25.6 \%$ to $10.6 \%$. Seventy-two patients were contacted prior to their PAC visit and a MedsCheck was completed for $73.8 \%$ (31/42) of eligible patients. From 15 patient surveys returned, all indicated interest in doing a MedsCheck in the future prior to surgery and agreed that MedsCheck helped them better understand their medications. From 12 pharmacist surveys returned, none indicated difficulty in scheduling a MedsCheck.

Conclusion: Our study demonstrated that integrating MedsCheck into our peri-operative process for orthopedic patients improved physician prescribing and facilitated pharmacist medication reconciliation while increasing patient satisfaction. Based on these results, we plan to implement this approach for all elective surgical patients at our institution.

\section{Evaluation of IV to PO Conversion Strategies in Surgical Areas}

Bernadette Chevalier', Heather Lummis', Tammy MacDonald?', Bev Mercer ${ }^{3}$, Lisa Martin', Patricia Ferguson ${ }^{4}$, Janice Williams ${ }^{4}$ ${ }^{1}$ Pharmacy Department, ${ }^{2}$ Health Services Manager $9 A,{ }^{3}$ Charge Nurse 9B, ${ }^{4}$ Charge Nurse 9A, Capital Health, Halifax, Nova Scotia

Rationale: Intravenous (IV) to oral (PO) conversion programs have resulted in efficacious treatment, fewer complications, shorter hospital stays, and cost savings. Drug utilization pharmacists worked with nursing staff of 2 surgical units to improve overall drug use and IV to PO conversion rates. One strategy proposed was the generation of daily IV medication lists on the wards for use by charge nurses.

Objective: To evaluate the impact of daily IV drug lists on IV to PO ratios, usage, and costs for targeted drugs.

Methods: Daily lists of patients on IV medications were scheduled to print on 2 surgical units beginning October 9, 2007. Charge nurses referred to IV lists to prompt IV to PO conversions on patient care rounds. Nursing units received educational information on IV to PO conversion. Evaluation methods included IV to $\mathrm{PO}$ ratios, drug usage (DDDs/100 beddays), and costs. IV to PO ratios and usage were compared for 6 months in 2006-07 to the same period in 2007-08. Drug costs were tracked from 2005-2008. Charge nurses were asked to provide feedback on the process.

Results: All targeted drugs showed a relative drop in IV usage compared to oral except fluconazole. The IV to PO ratio of ciprofloxacin decreased by $13 \%$. IV pantoprazole usage dropped sharply from 36.7 to 16.6 DDDs/100 beddays after the IV lists were introduced. Increased total drug costs for 2007-08 compared to 2006-07 were attributable to higher costs in the first half of 2007-08. Costs for targeted drugs decreased by $44 \%$ in the 6 months following the IV list initiative. Positive feedback was received from nursing and medical staff.

Conclusion: Using computer generated IV lists by charge nurses on patient care rounds appears to be an effective method in improving IV to PO conversion and in providing important patient information to nurses and physicians.

\section{Validating a Nurses' Satisfaction Survey about Their Perceptions of a Surgical Pharmacist}

Bernadette Chevalier', Heather Lummis', Kathryn Slayter' Claudia Harding', Anne Hiltz', Cheryl Leahy', Tammy MacDonald ${ }^{3}$ 'Pharmacy Department, 'Health Services Manager Thoracic Surgery, ${ }^{3}$ Health Services Manager General \& GI Surgery, Capital Health, Halifax, Nova Scotia

Rationale: The impact of clinical pharmacists $(\mathrm{CP})$ has been measured through interventions, acceptance rates, avoidance of adverse events, and associated cost savings as well as through the perceptions or opinions measured in surveys. Nurses' satisfaction surveys can help evaluate performance and identify expectations of clinical pharmacy services. An assessment of nurses' perceptions before and after the introduction of CPs on 2 general surgery wards was included as part of a formal evaluation of the new clinical pharmacy service.

Objectives: To validate the survey tool and to determine surgical nurses' perceptions of the clinical pharmacy service.

Methods: A survey was developed based on examples found in the literature. A 5-point Likert scale was used to rate 31 statements. Three categories included: performance evaluation (convenience, reliability, and accessibility), disconfirmation of expectations (service expected compared to service provided), and selfefficacy (CP impact on the nursing role). A power analysis of coefficient alpha indicated a sample size of at least 27 subjects was required to validate the survey. A factor and Cronbach's alpha analysis was used to determine reliability of the categories. Survey results were tabulated.

Results: Thirty surveys ( $93 \%$ of eligible staff) were completed by thoracic surgery nursing staff. All data were entered into a Microsoft Excel database for analysis. Cronbach reliability coefficients calculated for each category are as follows: performance (0.73), disconfirmation of expectations (0.78), and self-efficacy (0.72). Overall pharmacy service was rated favorably. Nurses' expectations of CPs emphasized drug therapy monitoring activities (77-93\%) and problem solving on the ward $(97 \%)$ over reducing drug costs (63\%). Nursing staff strongly felt a surgical CP would enhance their nursing role (87-93\%). These results were shared with pharmacy and nursing management.

Conclusion: All 31 questions were retained for the future surveys on general surgery wards. Nurses' responses identified key areas for pharmacy improvement.

\section{Evaluation of an Interdisciplinary Approach to Benzodiazepine Tapering in a Geriatric Day Hospital}

Barbara Farrell1,2,3, Lori Chen', Pamela Eisener-Parsche', Grant Russell2,3 Natalie Ward', Naomi Dore ${ }^{2}$

'Bruyère Continuing Care, Ottawa, Ontario; 'Élisabeth Bruyère Research Institute, Ottawa, Ontario; ${ }^{3}$ University of Ottawa, Ottawa, Ontario,

${ }^{4}$ The Ottawa Hospital, Ottawa, Ontario

Rationale: Although much is known about the clinical effects of long-term benzodiazepine use, few studies have explored the experience of frail elderly patients trying to reduce their dependence on these commonly prescribed drugs. This qualitative study aims to understand patient and provider experiences with benzodiazepine withdrawal in a Geriatric Day Hospital (GDH) setting, as a prelude to a future intervention.

Objectives: (i) identify components of successful interventions and processes that contribute to the care of GDH patients reducing benzodiazepine use, and (ii) gain an understanding of the experience of elderly individuals and of the interdisciplinary GDH team as they work with patients tapering benzodiazepines.

Study Design and Methods: Qualitative study using semi-structured interviews, input session, and chart review. Patients were aged 60 or older, currently using at least one benzodiazepine. Providers included GDH medical, allied health, nursing and staff members. Interviews and session were recorded, transcribed, and coded in NVIVO. Constant comparative analysis identified common themes, variations, and meanings.

Results: Thirteen providers participated in the input session and interviews. Provider themes included dynamic, patient-centred care, the belief that patients could stop benzodiazepines, and the socially supportive GDH environment. Knowledge about tapering varied among staff and many were unclear about their role in the process. Providers indicated more reliance on informal, verbal communication than on written chart documentation. Five patients consented to interviews. Emerging themes included experiences with daytime fatigue while using benzodiazepines, willingness to decrease use once aware of risks, and little difficulty stopping.

Conclusions: The GDH team vision and functioning seems to facilitate patients' ability to taper benzodiazepines, yet team members would like more role clarity. Patients are willing to try tapering, especially when side effects are explained. Future evaluation of an enhanced intervention should use ethnographic methods to observe patients and key providers.

Encore Presentation 


\section{Creation of a Research Assistant Position as a University of Waterloo Co-op Pharmacy Student Experience}

Barbara Farrell ${ }^{1,2,3}$, Tracy Gierman², Kelly Babcock

'Bruyère Continuing Care, Ottawa, Ontario; 'Élisabeth Bruyère Research Institute, Ottawa, Ontario; ${ }^{3}$ University of Ottawa, Ottawa, Ontario

Rationale: The introduction of a co-op pharmacy program provides different opportunities for students and employers but employers may be reticent to pay students for what they view as primarily a student learning experience. We present an approach to funding a student position that allows employers to gain and students to learn.

Description: We developed a pharmacy co-op student research assistant position affiliated with both our hospital pharmacy department and research institute.

Implementation: We proposed the student be involved in different stages of a variety of projects. Potential involvement at the stages of environmental scan/ literature review, research question development, data collection and analysis and knowledge transfer were identified. The institute funded the position understanding that funds from research projects benefiting from the student's involvement would be transferred to the central budget to cover salary cost. A job description was developed and posted on the University of Waterloo JobMine website; we participated in the interviewing (by videoconference) and matching process with two qualified applicants.

Evaluation: The research assistant was involved in aspects of seven ongoing projects. Outputs included qualitative theme analyses (2), quantitative data collection, analysis and summary reports (2), poster abstracts (3), proposal development (1) and draft publications (2). Salary funding was contributed by four projects. Pharmacist shadowing opportunities, within hospital and outpatient programs, plus other networking opportunities, were arranged.

Importance to Practice: Hiring a co-op pharmacy student with research interests was beneficial to our institution and to the student. Work was completed on several projects and initiated on other potentially fundable projects. The student benefited from exposure to both research and pharmacy practice at an early stage in her education. While important as an innovative approach to pharmacist recruitment, we also believe that this approach motivates and encourages young pharmacists to consider practice research as a viable career option.

\section{Hormone Replacement Therapy: An Analysis Focusing on Drug Claims by Female Seniors 2000 to 2007}

\section{Gaucher', J. Hunt ${ }^{2}$ \\ 'Canadian Institute for Health Information, Ottawa, Ontario, 2Canadian Institute for Health Information, Ottawa, Ontario}

Rationale: Hormone Replacement Therapy (HRT), including estrogen only (unopposed) and combination (estrogen plus progestin) regimens, has been used to manage menopause symptoms since the 1970s. Significant declines in HRT use were reported following publication of the Womens Health Initiative (WHI) study, which concluded that overall health risks exceeded the benefits of therapy. Objectives: This analysis was intended to identify trends in HRT use in female seniors on public drug programs in five Canadian provinces between 2001-2002 and 2006-2007.

Study Design and Methods: Claims level data from the National Prescription Drug Utilization Information System (NPDUIS) Database, were analyzed for female seniors on public drug programs in Alberta, Saskatchewan, Manitoba, New Brunswick and Nova Scotia. The analysis included calculation of the proportion of female seniors on these programs using HRT, and also examined trends in the dosage and type of therapy used.

Results: The rate of HRT use among female seniors in the five provinces dropped from $13.9 \%$ in $2001-2002$ to $5.2 \%$ in 2006-2007. The decrease in combination HRT use (24.9\% per year) was greater than the decrease in estrogen only HRT use $(14.7 \%$ per year). The vast majority of female seniors still using HRT in 2006-2007 were on estrogen-only regimens (84\%). Of women using the higher (0.625 mg) dose in 2001-2002 and still using HRT in $2006-2007,37 \%$ had switched to the lower $(0.3 \mathrm{mg})$ dose

Conclusion: This analysis provides insight into how new evidence affected HRT use in Canadian female seniors. The decline in HRT use observed was consistent with the results of other studies examining use during this time period. The majority of women still using HRT at the end of the study period were on estrogen-only regimens, with over a third of these women using a lower dose.

\section{Monitoring and Documentation of Outcomes from Targeted Interventions: An Interprofessional Quality Improvement Project}

Lawrence Jackson', Suzanne Plowman², Patricia Williams², Imelda Tablizo², Michael Matthews', Evelyn Williams', Alena Hung', Christine Li', Sonia Dyett', Victoria Hsu', Dean Yang', Froozan Amin', Edward Kung

Departments of Pharmacy', Nursing ${ }^{2}$ and Medicine; ; Aging \& Veterans Care, Sunnybrook Health Sciences Centre, Toronto, Ontario

Rationale: Pharmacists and other members of the health care team rely on nursing documentation related to medications to complete their assessments and provide optimal patient care. This project aimed to increase nursing documentation in the week after initiation of a medication by improving communication among nurses and providing nurses with a reference guide to help with selection of medication monitoring parameters.

Description of the Project: Infrequent nursing documentation of medication-related monitoring parameters in the Interprofessional Notes (IPN) subsequent to the initiation of medications was attributed to a lack of communication between nurses that a new treatment had been started and uncertainty regarding the parameters to monitor. Our strategy was aimed at increasing the frequency of nursing documentation related to medications.

What Was Done: For each new medication, nurses were requested to write a communication statement which included "date, issue, treatment, specific monitoring parameters and duration of monitoring" on the patient's Care Guide, a document that nurses refer to daily when developing their plan of care. Nurses could access several resources to generate monitoring parameters, including a newly created "Monitoring Guide for Common Clinical Conditions and Drug Therapy Outcomes". An interprofessional working group developed the study protocol, educated the unit staff and conducted a post-implementation audit.

Evaluation: A one-month audit of 23 charts, revealed 21 new medications in 19 patients. For $18 / 21(86 \%)$ of new medications there was a Care Guide communication statement. In 17/21 (81\%) of cases there was at least one entry in the week following the new medication order. In $8 / 17$ (47\%) of cases there were $\geq 2$ entries in the following week.

Importance to Practice: Through collaborative teamwork, a communication strategy was implemented and a resource guide for medication monitoring was created. This resulted in an increase in the frequency of nursing documentation of medicationrelated monitoring parameters.

\section{Can Pharmacy Students Effectively Partner with Pharmacists to Support Medication Reconciliation for Patients?}

Philip Lam, Morgan Harrison, Sara Ingram, Jason Volling, Michael Wong, Jin Huh, Gary Wong, Alejandro Montonya, Emily Musing, Olavo Fernandes

(1) University Health Network, Toronto, Ontario; (2) Leslie Dan Faculty of Pharmacy, University of Toronto, Toronto, Ontario; (3) Health Care Quality Residency Program, Tec de Monterrey -School of Medicine, Monterrey, Mexico

Rationale: Hospitals are searching for innovative strategies to effectively implement medication reconciliation practices and meet hospital accreditation requirements. This project aimed to develop, implement and assess a practice model for pharmacy students partnering with pharmacists in a front line role to support medication reconciliation activities for admitted patients in the emergency department (ED).

Description: Two third-year pharmacy students participated in a formal medication reconciliation education program that included a standardized patient evaluation and pharmacist observation. A systematic patient workflow was developed that outlined medication reconciliation activities for both the student and the pharmacist. This included independent patient interviews, completing best possible medication histories (BPMHs), admission reconciliation and discrepancy identification for admitted general medicine patients. Structured worksheets were created to aid in efficiently gathering patient information and to support the reconciliation process.

Evaluation: Over the course of 10 weeks, the two students completed over 303 patient BPMHs at two ED sites. For one site, the student assessed an average of 5.5 patients per day utilizing an average of 3.55 medication information sources to complete each $\mathrm{BPMH}$. The mean patient interview and mean reconciliation preparation/clarification times were $12.49 \pm 4.83$ and $12.75 \pm 5.34$ minutes respectively. Student contributions increased overall efficiency of the process, which allowed pharmacists in the area to focus on other pharmaceutical care activities. Students were also actively involved in supporting hospital-wide implementation activities linked to medication reconciliation including quality improvement data collection.

Conclusions: An effective model was developed and implemented for pharmacy students to systematically partner with pharmacists and patients in completing BPMHs and admission medication reconciliation. Students not only gained valuable clinical and patient interviewing experience, but actively contributed to patient care in a front-line role in the ED. 


\section{Computerized Prescriber Order Entry and Medication Reconciliation: Friends or Foes?}

Anna W. Lee', Jennifer Harrison', Bassem Hamandi', Yvonne Kwan', Clement Yuen', Tara Amos', Jin Huh', Gary Wong ',2, Kori Leblanc' Donna Williams', Kelly Lane', Tim Tripp', Peter G. Rossos, ${ }^{1,3}$, Dante Morra ${ }^{1,3}$, Peter Bray ${ }^{1,3}$, Scott Beattie ${ }^{1,3}$, Olavo Fernandes ${ }^{1,2}$

(1) University Health Network, Toronto, Ontario; (2) Faculty of Pharmacy, University of Toronto, Toronto, Ontario; (3) Faculty of Medicine, University of Toronto, Toronto, Ontario

Background: Patients admitted to hospital after surgery are particularly vulnerable to medication discrepancies. Computerized prescriber order entry (CPOE) systems may contribute to medication reconciliation challenges. Objectives of this study were to quantify and characterize home medication discrepancies for surgical patients in the $\mathrm{CPOE}$ environment without proactive reconciliation and then to use this information to design and implement a proactive multidisciplinary practice model.

Methods: All consecutive elective surgical patients at a tertiary care teaching hospital who had a preadmission clinic appointment were prospectively assessed for inclusion. The primary endpoint was to determine the number of patients with at least one unintentional home medication discrepancy at the time of postoperative admission. Discrepancies were characterized according to standardized categories and compared to a previous study conducted in a paper-based prescribing environment. Potential clinical impact of discrepancies was also assessed. Findings were then used to support the design of a multidisciplinary practice model.

Results: Between March 10 and April 2, 2008, a total of 394 patients were screened and 207 met inclusion criteria. Overall, $46.4 \%$ of patients had at least unintentional home medication discrepancy in the CPOE-based setting without proactive reconciliation compared to $37.9 \%$ in a paper-based $(n=214)$ setting $(\mathrm{p}=0.08)$. For CPOE, omissions were the most common type of medication discrepancy $(68.3 \%)$ and $32.9 \%$ of patients experienced at least one discrepancy with the potential to result in clinically significant harm. Compared to the paperbased setting, the CPOE environment appeared to eliminate certain discrepancy types such as illegible orders, misspelled drug names and orders for 'pharmacy to clarify'; however there was an increase in medication omissions.

Conclusion: Post-operative admission discrepancies related to home medications are still evident in the CPOE-environment without proactive reconciliation. Knowledge of the most common discrepancy types can facilitate the design implementation and optimization of a proactive medication reconciliation practice model.

\section{A Medication Reconciliation Tool \\ Georgina Mikhail, Alice Hogg, Karen McFarlane, Shellyna Moledina} Markham Stouffville Hospital Corporation, Markham, Ontario

Markham Stouffville Hospital Corporation's goal is to Make it Great! for patients and medication reconciliation is a top priority. Our admission process to complete a medication history and reorder patient's medications was inefficient and inaccurate as indicated by a 2002 pilot project. Changes were needed to streamline the process, reduce re-work by various disciplines and further improve patient safety. In September 2006, a documentation tool was created for use by nurses, pharmacists and physicians in the emergency department to capture the best possible medication history upon a patient's admission and also serve as a doctor's order sheet. Our intention is to improve patient safety by decreasing adverse events through timely reconciliation of patient's medications. Our goals are to decrease the rate of unintentional variances per patient, improve the documentation of intentional variances and improve our overall success index, which measures the effectiveness of the reconciliation process over time.

Comparing the 12 months post implementation of the medication reconciliation tool with the 8 months prior shows our average rate of unintentional variances decreased by at least $60 \%$, documentation of intentional variances improved by $80 \%$, and our overall success index improved by more than $50 \%$. Being proactive in obtaining the medication history before the physician orders the medications appears safer for the patient and more efficient for the staff. This directly translates to decreased medication errors, adverse events and unnecessary prolonged length of stay. We have refined our process to incorporate an electronic capture of data and submission. This poster will allow us to share our data and trends observed with the implementation of this valuable tool.

Encore Presentation

\section{Dofetilide is Safe and Effective in Preventing Atrial Fibrillation Recurrences in Patients Refractory to Other Antiarrhythmic Agents Accepted for Catheter Ablation}

Yana Shamiss, Atul Verma, Richard Oosthuizen, Denise Tunney, Bradley Sarak, Marianne Beardsall, Catherine Seabrook, Zaev Wulffhart, Bernice Tsang, Yaariv Khaykin

Southlake Regional Health Center, Newmarket, Ontario

Rationale: To assess the safety and efficacy of dofetilide in patients refractory to other anti-arrhythmic drugs (AAD), accepted for catheter ablation of atrial fibrillation (AF).

Background: Dofetilide is a Class III ADD, currently not on the Canadian market but it is available via special access program. Dofetilide has been associated with a high risk of Torsade de Pointes (TdP) and requires in-patient initiation, it is used as the last resort in refractory AF patients accepted for catheter ablation. A collaborative dofetilide education, initiation and dosing program was jointly developed by the Electrophysiology Program and the Department of Pharmacy at our institution. Safety and efficacy of dofetilide in those AF patients accepted for catheter ablation has not been previously assessed.

Methods: 127 patients (69\% male, 58\% paroxysmal, 60 \pm 10 years, AF duration $8 \pm 7$ years, failed $1.9 \pm 1.1 \mathrm{AAD})$ scheduled for catheter ablation of their AF between 02/2004 and 05/2008 received dofetilide. AAD were stopped 5 halflives pre-ablation ( 3 months for amiodarone). They were followed for $15 \pm 7$ months with success defined as no further symptomatic or asymptomatic $\mathrm{AF}$ ( $>30$ seconds) and partial success as a $50 \%$ reduction in AF burden.

Results: Thirty-three patients started dofetilide $158 \pm 167$ days before ablation. Nine had no improvement, 16 experienced a partial success, 6 had no further $\mathrm{AF}$ and 2 improved enough to forgo ablation. Immediately following ablation 68 patients started dofetilide, of which 14 had no improvement, 22 experienced partial success and 32 had no further AF. Twenty patients started dofetilide $119 \pm 153$ days post-ablation, of which 4 had no improvement, 7 experienced partial success and 9 had no further AF. Six patients stopped dofetilide during loading because of QT prolongation. No patient developed TdP.

Conclusions: Dofetilide appears safe and effective in preventing AF recurrences in patients refractory to other $\mathrm{AAD}$ accepted for catheter ablation.

\section{Pregabalin Associated Falls in an Elderly Patient with Mildly Reduced Renal Function}

Luc Simard', Anne Harley',2, Barbara Farrell ${ }^{1,2,3}$

'Bruyère Continuing Care, Ottawa, Ontario; ${ }^{2}$ University of Ottawa,

Ottawa, Ontario; ${ }^{3}$ Elisabeth Bruyère Research Institute, Ottawa, Ontario

Rationale: Pregabalin is approved for treatment of diabetes-related neuropathic pain and postherpetic neuralgia with increasing off label use for conditions such as spinal stenosis pain and post-operative pain in the elderly. We report on a case of falls in an elderly woman shortly after initiation of pregabalin.

Description: An 85 year old woman was started on pregabalin $75 \mathrm{mg}$ po bid for sciatica due to recent onset spinal stenosis. Following this she developed drowsiness, slurred speech, and fell 2 days later, suffering a hip fracture. While on a rehabilitation unit, she remained on pregabalin $25 \mathrm{mg}$ po tid as she felt it offered some pain relief. Her MMSE was 30/30, but she displayed decreased concentration and short term memory in conversation. Her urea was $6.6 \mathrm{mmol} / \mathrm{L}$, creatinine was $69 \mu \mathrm{mol} / \mathrm{L}$, and creatinine clearance was $56 \mathrm{ml} /$ minute (Cockcroft-Gault). Due to ongoing pain, her pregabalin dose was increased, and she fell from her wheelchair, sustaining a wrist fracture. She agreed to taper the pregabalin and start gabapentin for pain control. She reported improved concentration and physiotherapy reported marked improvements in her balance.

Analysis: Pregabalin is eliminated by renal excretion. The creatinine clearance indicates that dose adjustment could have been made with a suggested starting dose of $75 \mathrm{mg}$ per day. The Naranjo Probability Scale was 7 suggesting a probable adverse drug reaction. Her symptoms of slurred speech, drowsiness, falls and decreased concentration coincide with manufacturer-listed side effects. Many effects persisted even when pregabalin was given at reduced doses consistent with her creatinine clearance.

Importance to the Practitioner: The side effect profile of pregabalin may put elderly patients at risk of falling, even when dosed appropriately for renal function. Pharmacists and other health professionals should calculate creatinine clearance for patients using pregabalin, and adjust doses for even those with mildly reduced renal function. 


\section{Quality Improvement (QI) Program to Increase Adherence with Thromboprophylaxis Guidelines in Toronto Area Hospitals}

Geerts $W^{\prime}$, Diamantouros $A^{\prime}$, Papastavros $T^{1}, \cup D^{2}$

'Sunnybrook Health Sciences Centre, Toronto, Ontario; ${ }^{2}$ ISMP Canada, Toronto, Ontario

Rationale: Venous thromboembolism is the most common preventable complication in hospitalized patients and is associated with substantial morbidity, mortality and resource utilization. Despite the existence of effective thromboprophylaxis, audits demonstrate that prescribing rates are suboptimal. A recent systematic review revealed that multifaceted and interactive strategies are effective at enhancing adherence to thromboprophylaxis guidelines.

Objectives: Among 8 hospitals in the Greater Toronto Area (GTA), to assess: 1 . adherence with evidence-based thromboprophylaxis guidelines (ACCP Guidelines); and 2. whether a multi-component QI/knowledge translation toolkit will increase adherence with guideline-recommended thromboprophylaxis.

Methods: This project involves 3 phases. In the BASELINE PHASE, we recruited 7 community hospitals and 1 teaching hospital in the GTA and conducted an audit of recommended thromboprophylaxis use among 50 consecutive patients in 3 patient groups (general medicine, general surgery and hip fracture surgery) at each site. Using a cluster randomized design, the INTERVENTION PHASE has randomized hospitals to receive the multi-component intervention for one of the 3 patient group while continuing usual care in the other 2 groups. The intervention involves audit and feedback, use of preprinted orders, and pharmacy involvement. A second intervention cycle involves implementing the intervention in one of the other groups in each of the hospitals. After each cycle, a POST-INTERVENTION audit is conducted. This abstract presents the baseline audit data and describes the intervention strategy.

Results: In the baseline audit $(\mathrm{n}=1,175)$, rates of thromboprophylaxis were highest in hip fracture surgery with an average adherence of $79 \%$ (range, 21-95\%). Rates were lower in general surgery with an average of $43 \%$ (range, 15-84\%) and in general medicine with an average of 31\% (range, 13-65\%). Target rates for each group were $100 \%$. Adherence was patient-group specific rather than hospital-specific. The INTERVENTION PHASE is ongoing.

Conclusion: The results indicate that the "practice gap" between recommended thromboprophylaxis and clinical practice is an important patient safety problem. Since participating centres include teaching and community hospitals, our results are likely reflective of local and national practice.

\section{Drug Dosing Recommendations for Continuous Renal Replacement Therapy in Tertiary References: A Critical Appraisal}

Sean K. Gorman, ${ }^{1}$ Richard S. Slavik, ${ }^{2}$ Stefanie Lam³

${ }^{1}$ Clinical Services Unit - Pharmaceutical Sciences, Vancouver General Hospital, Vancouver, British Columbia; '2Pharmacy Department Interior Health Authority, Kelowna, British Columbia; ${ }^{3}$ Montreal General Hospital, McGill University Health Center, Montreal, Qubec

Rationale: A common drug-related problem in patients receiving continuous renal replacement therapy (CRRT) is suboptimal drug dosing. Clinicians commonly refer to tertiary drug information references to retrieve this information, however it is unknown whether these recommendations are complete and accurate.

Objectives: To determine the availability and accuracy of CRRT drug dosing recommendations in 4 drug information resources.

Methods: ICU quality assurance and pharmacy databases were searched to identify medications commonly prescribed during CRRT. A literature search was performed using PubMed, EMBASE, and International Pharmaceutical Abstracts to locate studies on drug dosing during CRRT for the identified medications, and evidencebased recommendations were developed. The Compendium of Pharmaceuticals and Specialties 2007 (CPS), the American Hospital Formulary System-Drug Information 2007 (AHFS-DI), Drug Prescribing in Renal Failure $4^{\text {th }}$ edition (DPRF $4^{\text {th }}$ ), and Drug Prescribing in Renal Failure $5^{\text {th }}$ edition (DPRF $5^{\text {th }}$ ) were searched for presence of CRRT drug dosing information. Doses recommended in these resources were compared to our evidence-based recommendations to determine accuracy.

Results: Evidence-based CRRT drug dosing recommendations were developed for 36 medications. The CPS and AHFS-DI did not provide dosing recommendations for any of the medications. DPRF $4^{\text {th }}$ and DPRF $5^{\text {th }}$ provided dosing recommendation for $72 \%$ and $83 \%$ of medications, respectively. Discordance with evidence-based dosing was observed for $31 \%$ and $13 \%$ of medications in DPRF $4^{\text {th }}$ and DPRF $5^{\text {th }}$, respectively. The dose was considered too low for $100 \%$ and $75 \%$ of the discordant recommendations in DPRF $4^{\text {th }}$ and DPRF $5^{\text {th }}$, respectively.

Conclusion: Two influential North American drug-information resources do not provide CRRT drug dosing recommendations. However, the 2 most recent editions of a specialty drug-information resource provide dosing recommendations for more than two-thirds of the medications examined with the latest edition being most accurate Accurate CRRT drug dosing recommendations should be provided in future editions of tertiary drug-information resources.

\section{Is Total Parenteral Nutrition with 4 and 5 Per Cent Amino Acid Solutions Safe?}

Dolores laboni, Andrea E Nash, Inez Martincevic, Susan Merko, Ali Estifaee, Robert P Jankov, and Todd Van Vliet

Neonatal Intensive Care Unit, Sunnybrook Health Science Centre, Toronto, Ontario

Rationale: Amino acid solutions more concentrated than the current maximum (3\%) in our unit are required to meet new parenteral protein recommendations for preterm infants on limited fluids. Theoretically, more concentrated protein solutions have higher solubility, though this issue has not been previously examined using Primene.

Objective: To examine for precipitates in batched 3, 4 and 5\% amino acid solutions containing commonly used additives, including calcium (Ca) and phosphorus (PO4). Methods: Solutions with varying amounts of protein (3, 4, $5 \%$ ), dextrose (5-12.5\%), $\mathrm{Ca}(9-20 \mathrm{mmol} / \mathrm{L}), \quad \mathrm{PO} 4(0-20 \mathrm{mmol} / \mathrm{L})$, electrolytes $(\mathrm{Na} 0-15 \mathrm{mmol} / \mathrm{L}, \mathrm{K}$ $0-6 \mathrm{mmol} / \mathrm{L})$, acetate $(0-4 \mathrm{mmol} / \mathrm{L})$ and a pediatric trace element mix were examined for precipitates up to 2 months after batching. Three 1 millilitre aliquots per sample (including positive controls known to contain Calcium Phosphate crystals) were visually screened using 6-well (35 mm diameter) cell culture plates and an inverted microscope using magnification 80 times. Association between each variable and precipitation was tested using logistic regression analysis.

Results: No "classical" CaPO4 crystals were found in any of the solutions examined. "Amorphous precipitates of unknown composition were observed in $6.1 \%(11 / 179)$ of bags examined. Precipitates were more frequently observed at higher $\mathrm{Ca}$ and PO4 concentrations (Table). There was no relationship between the presence of precipitates and the concentration of amino acids, glucose, or levels of electrolytes/acetate regardless of time point examined.

$\%$ Precipitation with varying concentrations of $\mathrm{Ca}$ and PO4 \begin{tabular}{llll}
\hline $\mathrm{Ca}(\mathrm{mmol} / \mathrm{L})$ & $\%$ with precipitates & $\mathrm{PO} 4(\mathrm{mmol} / \mathrm{L})$ & $\%$ with precipitates
\end{tabular}

\begin{tabular}{lccc}
\hline- & - & 0 & 0 \\
9 & 0 & 9 & 0 \\
15 & 9.2 & 15 & 9.2 \\
20 & 11.4 & 20 & 11.4 \\
\hline Associations: Ca and precipitate $\mathrm{P}=0.0143, \mathrm{PO}$ and precipitate $\mathrm{P}=0.0161$
\end{tabular}

Conclusion: The possibility of precipitation (and need for routine filtering of TPN) should be considered in high $\mathrm{Ca}$ and PO4 solutions, irrespective of the concentrations of the other components, including amino acids.

\section{The Guideline to Assess Pharmacotherapy Scale (GAPS): Preliminary Data}

Jamie Kellar, ${ }^{2}$ Saulo Caste ${ }^{1,2,4}$, David Streiner ${ }^{1,3}$, Monica Scalco ${ }^{1,2,3}$

'Dept. of Psychiatry, University of Toronto, Toronto, Ontario;

'Whitby Mental Health Centre, Whitby, Ontario,

${ }^{3}$ Baycrest Centre for Geriatric Care, Toronto, Ontario;

${ }^{4}$ Sunnybrook Health Sciences Centre, Toronto, Ontario

Rationale: An unprecedented number of evidence based guidelines for pharmacological treatment of psychiatric disorders have been published in the last decade; few have been accompanied by strategies to assess compliance.

Objective: The authors developed the Guideline to Assess Pharmacotherapy Scale (GAPS) for the assessment of pharmacological treatment of psychiatric disorders.

Methods: The GAPS was developed and pilot test was performed by independently scoring 89 discharges from an inpatient unit of a tertiary psychiatric hospital, comparing the prescribed medication(s) with the available national guidelines for the condition(s) diagnosed at discharge. Six senior care providers from different institutions were presented the scale and provided input.

Results: The final version of the GAPS appraises 6 variables related to the treatment(s): (1) The strength of the evidence supporting it (zero for first line recommendation, 1 point for second line, etc.); (2) the number of prescribed medications (zero for adequate and 1 point for each extra or missing medication); (3) the types of prescribed medications (zero for adequate and 1 point for each extra or missing medication); (4) the dosage of the prescribed medications (1 point for dosages under the recommended range and 2 points for dosages above); (5) the prescription of proscribed medications ( 4 points each); and (6) untreated identified conditions (1 point for each). The total score ranges from zero to unlimited, and the lower the score, the stronger the evidence to support the treatment. The same 89 discharges were scored with the final version, again independently by 2 authors. The scores ranged from 0 to 10 , mean 2.3 and standard deviation 1.9.

Conclusions: The GAPS provides a more detailed assessment of closeness to the guidelines than a dichotomous yes/no scoring system. To our knowledge, this is the first scale to score pharmacological treatments by comparing them to available guidelines. 


\section{Topiramate-Induced Hemiplegia}

Joel Lamoure, Jessica Stovel

London Health Sciences Centre, London, Ontario

Rationale: Topiramate is officially indicated for epilepsy and migraine prophylaxis, but is also used as a mood stabilizer as it is weight negative. Many side effects observed are dose-related and result from too rapid dose titration. While paresthesias are thought to be dose-related and occur in $50 \%$ of patients exceeding doses of $100 \mathrm{mg} /$ day, there is no mention of hemiplegia in the product monograph and only 2 cases of hemiplegia are reported in the literature.

Description: A 36-year old female presented to hospital with left-sided weakness, facial droop, slurred speech, tremors, drowsiness, decreased cognitive function, dizziness, shuffling gait, recent history of falls, and flat affect. Symptoms had developed starting three weeks prior to admission. The patient's medical history is significant for schizoaffective disorder, depression, epilepsy, and a heart murmur. Medications on admission included: topiramate, zuclopenthixol benzotropine, lamotrigine, clonazepam, and citalopram. One month prior to admission, the patient's topiramate dose had been increased to $100 \mathrm{mg}$ daily.

Assessment of Causality: Upon admission, a temporal-spatial relationship between symptoms and the dose increase of topiramate were hypothesized by the pharmacist and physician. The patient's symptoms showed marked improvement with a topiramate dose reduction over 6 days and complete resolution at discontinuation. The Naranjo Probability Scale yields a score of 7 , suggesting a probable adverse drug reaction. There was no re-challenge of topiramate.

Evaluation of the Literature: There are only two case reports of topiramateinduced hemiplegia in the literature. Both patients had pre-existing compromised neurological function, but presented similarly to our patient. After withdrawal of topiramate, symptoms completely resolved within either two or eight weeks in the literature.

Importance to Pharmacy Practitioners: Recognition of this adverse reaction is important because it may help prime pharmacists that topiramate may be a cause of hemiplegia and withdrawal of topiramate may be indicated in these patients.

\section{Mortality in Febrile Neutropenic Patients Treated Initially with Cefazolin and Tobramycin}

Francesca Le Piane ${ }^{1,2}$, Sandra Walker ${ }^{1,2}$, Scott Walker ${ }^{1,2}$, Nina Lathia ${ }^{1,2}$ Carlo De Angelis, ${ }^{1,2}$,Andrew Simor ${ }^{3,4}$

'Sunnybrook Health Sciences Centre, Department of Pharmacy, Toronto, Ontario; '2University of Toronto, Leslie L. Dan Faculty of Pharmacy, Toronto, Ontario; ${ }^{3}$ Sunnybrook Health Sciences Centre, Division of Infectious Diseases, Toronto, Ontario; ${ }^{4}$ University of Toronto, Faculty of Medicine, Toronto, Ontario

Rationale: Several antimicrobial regimens are considered suitable for the empiric management of febrile neutropenia. At Sunnybrook Health Sciences Centre, the recommended empiric regimen has been Cefazolin and Tobramycin for at least 25 years. We however, had no objective data to reassure us that patient mortality has not increased over the previous 5 years.

Methods: We conducted a retrospective chart review of 48 episodes occurring in 44 patients admitted for febrile neutropenia secondary to chemotherapy, initially managed with Cefazolin and Tobramycin in 2002. Prospective data from 48 episodes in 2007 had previously been collected. Data regarding patient demographics, cancer diagnosis, chemotherapy, risk score, antibiotic use, infection and episode resolution was compared. The primary objective of this study was to compare the all-cause mortality in 2007 to 2002.

Results: There were no statistically significant differences between the groups ( $\mathrm{p}>0.05)$. All cause mortality in 2007 was $8.3 \%$ (4/48) compared with $10.4 \%$ $(5 / 48)$ in $2002(\mathrm{p}=1)$. The sample size of 48 episodes per group had a power of $80 \%$ to detect a rise (one-tailed) in mortality of $7.4 \%$ (upper one-tailed limit of $95 \%$ confidence interval is $17.8 \%$ mortality) at an alpha of 0.05 and incidence of mortality in 2002 of $10.4 \%$. All deaths occurred in patients considered high risk according to the Talcott score.

Conclusion: Mortality has not increased in the last 5 years with the use of empiric Cefazolin and Tobramycin for the treatment of febrile neutropenia at our hospital and is comparable to rates reported in the literature for similar patients with febrile neutropenia. The results of this study provide reassurance that the regimen continues to be effective for our febrile neutropenic patients.

\section{Evaluation of Outcomes in Patients who Received Activated Protein C (Drotrecogin alfa [Activated]) in an Intensive Care Unit: A Retrospective, Observational Study}

Erika I.A. Maher, ${ }^{1}$ Barbara L. Falkner, ${ }^{2}$ Tammy D. Bluemink,

Amanda C. Shea, ${ }^{3}$ Cathy A. Antoniazzi ${ }^{4}$

'Pharmacist, Saint John Regional Hospital, Saint John, New Brunswick. ${ }^{2}$ CU Clinical Pharmacist, Northern Health, Prince George, British Columbia; ${ }^{3}$ Clinical Pharmacist, Northern Health, Prince George, British Columbia; ${ }^{4}$ CU Charge Nurse, Northern Health, Prince George, British Columbia

Rationale: The pathophysiology of sepsis primarily involves coagulation, inflammation and impaired fibrinolysis, which is responsible for the high morbidity and mortality rates of severe sepsis and septic shock. Drotrecogin alfa (activated) [DAA], a recombinant form of activated protein $\mathrm{C}$, has been associated with a reduction in mortality but is costly and associated with an increased risk of serious bleeding.

Objectives: The objectives were to describe clinical outcomes (mortality, bleeding) of patients who received DAA and compare the results to those in the current literature, to describe patient and treatment characteristics and evaluate if any serve as possible predictors of mortality, and to evaluate compliance with the institutional order form for the use of DAA.

Methods: This retrospective, observational study involved chart reviews and a literature search. Patients who received DAA at Prince George Regional Hospital from January 2003 to November 2007 were included. Data collected included demographics, medical history, infection data, labs, illness severity, DAA treatment data, outcomes (mortality, bleeding), and adherence to the order form. Descriptive statistics were used to summarize the data and compare results to previous studies.

Results: Nineteen patients were included, demonstrating a mortality rate of $58 \%$ compared to $24.7 \%-45.2 \%$ in the literature, and a serious bleeding event rate of $11 \%$ as compared to $3.5 \%-7.3 \%$ in the literature. Patient characteristics that suggested a trend toward increased mortality included $\geq 3$ organ failures, respiratory system failure and infection with Candida. Compliance with the order form was $100 \%$ for inclusion criteria, $95 \%$ for exclusion criteria.

Conclusion: The results of this small study demonstrated higher mortality and serious bleeding rates as compared to the literature, while adherence to the order form was high. Until more RCT's are available to provide more robust efficacy and safety data, observational studies will help guide our practice regarding the use of DAA.

\section{Assessing the Clinical Usefulness of Checking Postprandial Glucose Levels when Fasting Glucose Levels are Near Normal in Patients with Type 2 Diabetes}

Kerry D Mansell,' David F Blackburn,' Dean T Eurich

'College of Pharmacy and Nutrition, University of Saskatchewan, Saskatoon, Saskatchewan; ${ }^{2}$ School of Public Health, University of Alberta, Edmonton, Alberta

Rationale: Self-monitoring of blood glucose (SMBG) is typically recommended to patients with type 2 diabetes, in part, to facilitate achievement of both fasting and postprandial glucose (PPG) targets. However, considering the significant costs associated with SMBG coupled with the fact that the optimal timing/frequency of SMBG is unknown, it is unclear if patients should be instructed to routinely monitor postprandial levels, especially when fasting plasma glucose (FPG) targets are being achieved.

Objective: To determine if PPG testing provides additional important clinical information beyond FPG tests in well-controlled type 2 diabetes patients.

Methods: Consenting subjects with type 2 diabetes that tested FPG levels $\geq 3 \mathrm{x} /$ week, had FPG levels $\leq 8.0 \mathrm{mmol} / \mathrm{L}$ on $75 \%$ of tests, and measured PPG $\leq 4 \mathrm{x} /$ month were eligible. Subjects were recruited from 8 pharmacies and instructed to perform daily FPG tests during Days 1 to 7 (run-in phase), and daily FPG and PPG tests during Days 8 to 21 (test phase). Descriptive statistics were used to summarize FPG and PPG levels. For phase 2, McNemar's test for paired data was used to valuate the proportion of tests within both FPG and PPG target ranges.

Results: The mean (SD) FPG from 362 tests ( $\mathrm{n}=52$ subjects) in the run-in phase was $7.0 \mathrm{mmol} / \mathrm{L}$ (1.4). During the test phase, mean FPG was also $7.0 \mathrm{mmol} / \mathrm{L}$ (1.6) and the mean PPG was $8.4 \mathrm{mmol} / \mathrm{L}$ (2.2). Among all the instances where FPG was $<7.0 \mathrm{mmol} / \mathrm{L}$ during the test phase, $87.7 \%(322 / 370)$ of corresponding (same day) PPG tests were $<10.0 \mathrm{mmol} / \mathrm{L}$. Among individuals with mean $\mathrm{FPG}<7 \mathrm{mmol} / \mathrm{L}$ (29/51), $87.4 \%$ of all PPG tests were also within recommended limits $(<10.0 \mathrm{mmol} / \mathrm{L})$.

Conclusions: The results of this pilot study suggest that in type 2 diabetes patients with controlled fasting glucose levels, daily PPG testing likely provides only limited additional clinical information for patients or clinicians; however, further study is required. 


\section{Epoprostenol-Associated Thrombocytopenia Associated with Clinical Bleeding}

N. Parker, C. Bayliff, S. Mehta, B. Bunting

London Health Sciences Centre, London, Ontario

Rationale: : Epoprostenol (EP) is used for the treatment of pulmonary arteria hypertension (PAH). Generally EP produces side-effects related to vasodilation, including flushing and headache, which can be minimized by gradual dosage titration. EP also has antiplatelet effects but only infrequently results in thrombocytopenia. We describe a case report of EP-associated thrombocytopenia accompanied by clinical bleeding.

Description: A 38-year-old female with WHO/NYHA Class III PAH associated with congenital heart disease was admitted to hospital to begin EP after failing bosentan thearapy. The patient was initiated on EP $2.1 \mathrm{ng} / \mathrm{kg} / \mathrm{min}$. At this time, her platelet count was $146 \times 10^{\circ} / \mathrm{L}\left(\mathrm{N} 150-400 \times 10^{\circ} / \mathrm{L}\right)$. EP was increased to $3 \mathrm{ng} / \mathrm{kg} / \mathrm{min}$ on day 2 with subsequent worsening of thrombocytopenia. On day 4 she complained of mild nasal bleeding and on day 7 she began to experience vaginal spotting.

Assessment of Causality: Though disease process may also play a role in patients who develop thrombocytopenia, given the rapidity of decline of platelets upon initiation of EP, it is probable that EP played a role in her thrombocytopenia. The Naranjo causality scale indicates a probable ADR with a score of 6 .

Evaluation of the Literature: EP has been associated with functional thrombocytopenia through its antiplatelet effects but actual reduction in platelets is uncommon. Reports of EP-associated thrombocytopenia have been documented.

Importance to Pharmacy Practitioners: Pharmacists should be aware of the possibility of clinically important thrombocytopenia in association with EP therapy for $\mathrm{PAH}$.

\section{Development and Implementation of a Pharmacy Mentorship Program}

Salma Satchu, Elaine Tom, Christinne Duclos, Kathy Vu St. Michael's Hospital, Toronto, Ontario

Rationale: Professional growth and development in terms of practice-related experiential mentoring was identified from a departmental retreat as a priority need amongst pharmacists. Therefore, the objective of this project was to create and pilot a mentoring program for pharmacists.

Description of Project: A needs assessment of professional growth of the pharmacists was conducted via anonymous survey, which led to the development of a pilot mentorship program. Barriers that were addressed included resources for mentoring, defining expectations of the roles of mentor and mentee, and the need for a key person to facilitate various logistics such as mentee-mentor matching, scheduling, and objectives development. . Two models were developed to address various needs: task-directed (where meetings are focused on specific topics and clinical activities), and collaborative patient care related (where mentee provides care to patients with mentor guidance).

Evaluation: The pilot resulted in completion of 5 successful pairings with 11 pharmacist participants (one pairing had 2 mentees and 1 mentor). Evaluation surveys were completed by both mentors and mentees upon completion of the program, which included assessment of the mentoring relationship and the program logistics. All mentees and mentors reported high levels of satisfaction with matching and mentoring relationship. All mentees and mentors felt that the mentoring relationship facilitated achievement of objectives.

Importance: The success of the pilot resulted in subsequent development a formal departmental mentorship program. One mentee who completed the formal program thus far was successfully recruited into the clinical practice area of the mentor, exemplifying how this type of program can promote professional growth and aid in retention of staff.

\section{Development of a Chart Audit Process to Facilitate Pharmacists' Documentation}

Elaine Tom, Diane Chong

St. Michael's Hospital, Toronto, Ontario

Rationale: Pharmacists' documentation, while important in broadening the communication network of patients drug related issues, is not always consistently performed given time constraints. The purpose of this project is to audit charts for the presence of documentation, adherence to St. Michael's Hospital documentation policies, and to provide feedback to pharmacists to encourage and improve documentation practice.

Description of Project: A pharmacy data collection tool was developed based on existing tool used by other health disciplines. From June 2007, chart audits were conducted by a pharmacist on a quarterly basis. Charts were randomly selected by Health Records, varied by service and unit, with each audit encompassing 44-112 charts depending on availability. The data collected included the number and content of notes in each chart. To measure the quality of the documentation, content was compared to legal and departmental policy requirements.

Evaluation: Five quarterly audits totalling 362 charts have been conducted. The number of charts audited where pharmacy note(s) were present increased from $11(24 \%)$ to $49(44 \%)$ during each quarter period. A progressive increase was seen in the total numbers of pharmacy notes written ranging from 16 (first quarter) to 83 notes (last quarter). Results of each audit were shared with pharmacists. General and individual feedback was provided to the pharmacists to improve the quality of the documentation practice. Feedback of data to the pharmacists, simplified forms, electronic note, and documentation tutorials have been implemented regularly to address some of the reported barriers.

Conclusion: Chart audits provided valuable information in current state of practice and barriers which formed basis of improvement interventions. In addition, this information is necessary to facilitate our transition to electronic documentation and to our goal of complete documentation.

\section{Systemic Reaction Associated with Nitrofurantoin Presenting as Leukocytoclastic Vasculitis}

S. Wong, C. Bayliff

London Health Sciences Centre, London, Ontario

Rationale: Nitrofurantoin is used for the treatment of urinary tract infections. While pulmonary vasculitis is not uncommonly reported, cutaneous vasculitis with pulmonary involvement is rarely seen. We report a case of systemic reaction to nitrofurantoin presenting as leukocytoclastic vasculitis (LV).

Description: An 85-year-old female presented with a 3 day history of petechial maculopapular rash on both tibial aspects of lower extremity. Five days earlier she had been commenced on nitrofurantoin. The non-pruritic and non-blanching rash extended superiorly to knee, and distally to dorsum of foot. The patient described the rash as painful and burning in nature. She also complained of dyspnea. Crackles were noted in the left pulmonary lobe, and had her oxygen saturation measured at $91 \%$ on room air. On examination, patient was afebrile, hypertensive with blood pressure of $160 / 76 \mathrm{~mm} \mathrm{Hg}$, tachypneic at 28 breaths/minute, and tachycardic at 96 beats/minute. Abnormal laboratory values indicated elevations in eosinophil $\left(1.1 \times 10^{\%} / \mathrm{L}\right), \operatorname{ESR}(72 \mathrm{~mm} / \mathrm{h})$, and CRP $(66 \mathrm{mg} / \mathrm{L})$. Further investigations revealed diffuse parenchymal lung disease. Discontinuation of nitrofurantoin resulted in improvements in dyspnea, purpuric rash, oxygenation, and ESR $(54 \mathrm{~mm} / \mathrm{h})$.

Assessment of Causality: Application of Naranjo score of 6 suggested that this was a probable $\mathrm{ADR}$. Classic presentation of cutaneous vasculitis involves burning, erythematous macules, purpura, fever, and myalgia, occurring 7-21 days after initiation of nitrofurantoin. Pulmonary hypersensitivity presents as dyspnea, tachypnea, tachycardia, pulmonary edema, eosinophilia, and increased ESR.

Evaluation of the Literature: While hypersensitivity reactions to nitrofurantoin are not infrequently reported combined cutaneous vasculitis and pneumonitis caused by nitrofurantoin is rarely reported.

Importance to Pharmacy Practitioners: Pharmacists need to be aware of the potential of nitrofurantoin to cause severe adverse effects.

Encore Presentation 


\section{Development of an Evidence Based Handbook for Healthcare Providers on Exposure to Psychotropic Medications and Other Substances During Pregnancy and Lactation}

Wende Wood', Adrienne Einarson'2, Betty Dondertman 'Centre for Addiction and Mental Health, Toronto, Ontario, 'The Motherisk Program, Toronto, Ontario

Rationale: Lack of information and misinformation exists regarding women's use of drugs during pregnancy and lactation. A health care provider's challenge is to understand the evidenced-based benefits and risks, both to the mother and to he fetus or baby, of taking versus discontinuing the use of psychotropic medications and other substances.

Description of Service and Steps Taken to Develop and Implement New Program: A proposal was submitted to the Health Canada Drug Strategy Community Initiatives Fund, resulting in a 3 year financial award. A multidisciplinary team from across Canada wrote and reviewed several drafts which resulted in the 115 page book entitled: Exposure to Psychotropic Medications and Other Substances during Pregnancy and Lactation: A Handbook for Health Care Providers. The handbook details the properties and effects of psychotropic medications and other substances, and provides recommendations on how to advise women using these substances. Common myths regarding drug exposure during pregnancy and lactation are discussed, as well as key principles for clinical approaches to working with pregnant or breastfeeding women. Quarterly live webinars to keep up to date with new evidence based information are enhancing the knowledge transfer portion of the project. Participants in these webinars also have access to a message board community to further share information and experiences.

Evaluation of Project: A post-webinar survey is on Survey Monkey for participants to evaluate the book and webinars.

Importance and Usefulness to Current and Future Practice: Misinformation and stigma about using psychiatric medications and other substances during pregnancy and a lack of easily accessible evidence based information can lead to women receiving inadequate or even no prenatal care. With the use of this handbook, health care providers will be better equipped to counsel their patient on these issues. This will help ensure the best outcome possible for both mother and baby, as the woman will have a higher likelihood of good mental health.

\section{Case Report: Sibutramine-Associated Unstable Angina}

Katherine Bateman, Charles Bayliff, Kumar Shridar London Health Sciences Centre, London, Ontario

Rationale: Obesity is associated with substantial cardiovascular morbidity and mortality and these risks may be decreased by weight loss. Sibutramine is indicated in obesity for weight loss; however, sibutramine may independently contribute to adverse cardiovascular outcomes. We describe a case of sibutramineassociated unstable angina.

Description of Case: A 60-year-old $100 \mathrm{~kg}$ man was transferred to our hospital with a diagnosis of unstable angina. Two days earlier he had presented to another Emergency Room with complaints of headache, nausea, and chest discomfort (relieved in hospital with nitroglycerin). His troponin-I was $0.1 \mu \mathrm{g} / \mathrm{L}$ (normal $\leq 0.8$ ) and he had minor T-wave abnormalities on his electrocardiogram. This patient had a 144 pack-year history of smoking prior to quitting two weeks before, type 2 diabetes mellitus, hypertension, peripheral vascular disease with symptomatic claudication in the legs, hypercholesterolemia, obstructive sleep apnea, osteoarthritis in the knee, and obesity, for which he had started treatment with sibutramine $10 \mathrm{mg}$ once daily within the past 2 weeks; he had no prior history of angina. Coronary catheterization showed widespread mild small vessel disease without significant narrowing and no major vessel disease. The patient was treated medically and subsequently discharged home with directions to not restart sibutramine.

Assessment of Causality: Cardiovascular adverse effects of sibutramine include increased blood pressure, heart rate, and angina. Temporally, sibutramine appeared to play a role in this patient at high risk of cardiovascular disease. A score of 2-3 on the Naranjo Causality Scale suggested a possible reaction.

Evaluation of Literature: There have been several reported cases of angina caused by sibutramine, as well as a case report of myocardial infarction associated with sibutramine in an otherwise healthy 24-year-old woman.

Importance of Case to Pharmacy Practitioners: Pharmacists should be aware of cardiovascular risks associated with commencing anti-obesity agents.

\section{Analysis of Patient Controlled Analgesia and Oral Step-Down Therapy for Quality Improvement in Pain Management}

\section{Robin Brockhouse, Rosemary Tanzini}

St. Michael's Hospital, Toronto, Ontario

Rationale: A quality improvement initiative is underway for patient satisfaction regarding pain management - especially for pain following orthopedic surgery. Before improvements can be made, the problem areas in pain management need to be identified. The ordering and usage of patient-controlled analgesia (PCA) and ordering of step-down therapy were identified as possible problem areas.

Objectives: This study aimed to describe patterns related to the ordering and usage of PCA and ordering of step-down therapy for the purpose of quality improvement in pain management.

Study Design and Methods: The pharmacy information system was used to generate a list of all PCA orders during a 2-month period (April - May 2008). A subset of patients was selected from this list for retrospective chart review in an attempt to produce a sample which roughly replicated the overall proportions of opioid type and service using PCA. Chart review was performed using scanned charts available electronically. Standard data sheets were used to manually record data. The PCA used as a percentage of PCA available to the patient was calculated. Equianalgesic doses were calculated and compared for PCA doses used in the 24-hour period before discontinuing PCA versus step-down dosing ordered for the first 24 hours after discontinuing PCA.

Results: Of 508 episodes of PCA usage, a representative sample of 27 patients were selected for chart review. Patients used $28 \%$ of their allowed dose of PCA; orthopedics patients used $21 \%$ while patients from other services used $29 \%$. A typical step-down order of oxycodone $5 \mathrm{mg} /$ acetaminophen $325 \mathrm{mg} \mathrm{1-2} \mathrm{tablets}$ q4h was ordered most frequently (13/27 cases). Step-down orders were dosed higher than previous PCA usage in 11/27 cases, dosed lower in 9/27, and dosing ranges overlapped in $11 / 27$ cases.

Conclusion: The data revealed that patients were not maximizing their use of PCA. Additionally, step-down dosing frequently differed from the prior PCA usage. Dosing of step-down therapy was not always inadequate; doses were higher than necessary at least as often as they were lower.

\section{Use of the CSHP 2015 Self-Assessment Survey to Determine Progress toward CSHP VISION 2015}

\section{Naomi Dore', Kelly Babcock², Barbara Farrell',2,3 \\ 'Élisabeth Bruyère Research Institute, Ottawa, Ontario; \\ ${ }^{2}$ Bruyère Continuing Care, Ottawa, Ontario; \\ ${ }^{3}$ University of Ottawa, Ottawa, Ontario}

Rationale: To evaluate our progress towards CSHP VISION 2015 via input from pharmacists and management.

Description: Fifteen pharmacists from palliative care, rehabilitation, complex continuing care, family health team, long-term care programs and management, individually completed the standard self assessment survey.

Steps Taken: Survey answers were collated by programs and management and presented graphically to staff. Strengths and gaps were discussed.

Evaluation: Responses demonstrated strong performance in activities related to direct patient care and integration of pharmacists, (e.g. medication reconciliation, monitoring of complex, high-risk medications, collaboration with team and evidence-based care provision) consistently across programs. The main gap perceived was in the area of technology use, confirming results of a previous Failure Mode and Effects Analysis conducted on all medication related practices. There were different interpretations of performance in some areas between programs and by management (e.g. safety initiatives, technician certification, smoking cessation counseling). The survey tool seemed to lack specificity to our non-acute care institution (e.g. Goal 3 question wording did not highlight strengths in ensuring evidence-based care provision because patients are not hospitalized specifically for acute MI and most interventions occur before discharge). Clarity of some questions was discussed (e.g. definitions of unit-dose and understanding of clinical decision support). Discussion ensued regarding the value of residency programs.

Usefulness to Practice: We used this data to formally establish the status of our progress towards CSHP 2015 with input from all pharmacists. We developed action plans to move forward on our perceived weaknesses in the area of technology, some patient safety issues and in communication to ensure all pharmacists and management are aware of each others best practices. The survey results highlighted our strength of pharmacist integration into the health care team. 


\section{PPC 2009 POSTER ABSTRACTS / RÉSUMÉS DES AFFICHES DE LA CPP 2009}

\section{Medication Administration Record Action Review \\ John Dos Santos, Diana Shin, Sue Kaczmarczyk, Lisa Vandermolen, Lynne Kelly, Barbara Watterworth, Anne Marie Bombassaro London Health Sciences Centre, Victoria Hospital, London, Ontario}

Rationale: Medication administration record (MAR) actions are discrepancies identified by nursing on the pharmacy generated medication profile. A hospital wide assessment of their content was undertaken as a means to identify potential safety issues and the need for improving departmental procedures.

Description: A prospective review of MAR actions received by pharmacy was performed. The objectives were to standardize internal review procedures, determine the number and types of issues identified and the time required for resolution.

Steps: MAR actions were collected for 21 consecutive weekdays. Two dedicated pharmacy technicians performed chart review for each MAR action. The tota time required was recorded on a daily basis. MAR actions were categorized independently by the technicians and 2 pharmacy students and by consensus with a pharmacist according to predefined criteria.

Evaluation: There were 58,666 prescriptions processed by pharmacy which resulted in 1238 MAR actions (2.1\%), of which 1230 were evaluable. The 2 mos common categories were order entry errors $(32.8 \%)$ and modification requests $(30 \%)$. Order entry errors were characterized mainly by drug omissions $(35.2 \%)$, failure to discontinue/suspend therapy $(27.8 \%)$ and incorrect frequency $(12.9 \%)$ Analgesics, anti-emetics, sedatives and antibiotics were most commonly implicated. The majority of modification requests $(71.8 \%)$ involved changes in the time or day of drug administration. Only 3/1230 evaluable MAR action were concurrently submitted by nursing to risk management. MAR action procedures required approximately 3 hours per day overall of technician time.

Relevance: The standardized MAR action review process was successfully managed by pharmacy technicians with minimal pharmacist involvement. MAR actions represented a small percentage of total orders, but consumed a considerable amount of time for resolution. Order entry discrepancies constituted the largest category of MAR actions and often involved high risk drugs, raising the need to investigate recertification procedures. Occurrence reports submitted to risk management under-represent potential medication safety issues.

\section{Visual Aids Enhance Inpatient Mental Health Education}

Sonia Dyett, Melissa Conte

\section{Sunnybrook Health Sciences Centre, Toronto, Ontario}

Rationale: A once weekly, hour long, pharmacist-run medication education group is part of an interdisciplinary group program in an acute care 35-bed inpatient psychiatric unit. Traditionally the group has used a question-and-answer format along with unstructured teaching around various medication issues. This group activity aims to improve patient's understanding and management of psychiatric medications.

Description of Project: It was felt that learning, understanding, and retention of medication related knowledge could be improved with visual aids and these would provide a more structured framework for the pharmacist's teaching. Drawing on the pharmacist's past experiences with the group, a series of 6 large posters were developed to better illustrate concepts and provide information for the patients. Content included names, classes and purposes of psychiatric medications, diagrams of how medications exert their effects in the brain common side effects and how to manage them, graphs to illustrate concepts of therapeutic range and steady state, tips on being adherent to medications, and miscellaneous tips on medication management (precautions, drug interactions, storage, etc.)

Evaluation: A simple evaluation form was developed to elicit feedback about the effectiveness of the posters as well as overall satisfaction with the group. Ideas will be incorporated into future posters, and general suggestions are being utilized to improve the focus and style of group discussions.

Importance to Practice: Psychiatric illnesses are associated with a low rate of adherence and a high rate of relapse. Knowledge of medication issues can lead to increased adherence and safer use of medications upon discharge. Improving the group learning experience can ultimately enhance medication adherence and safety upon discharge, and contribute to the patient's overall wellness.

\section{Developing Strategies to Improve Medication Reconciliation on Hospital Admissions}

Philip Lam ${ }^{1,2}$, Jin Huh', ${ }^{1,2}$, Michael Wong ${ }^{1,2}$

(1) University Health Network, Toronto, Ontario; (2) Leslie Dan Faculty of Pharmacy, University of Toronto, Toronto, Ontario

Rationale: Admission Medication Reconciliation (AMR) is performed by comparing the Best Possible Medication History (BPMH) with admission orders. At Toronto Western Hospital, pharmacists are trained in documenting this process using the Electronic Medication Information Transfer Tool (EMITT).

Description: The aim of this project was to measure the proportion of patients with a BPMH and AMR completed on admission, to examine how different entry points of admission affect this process, and to develop strategies to improve this measurement.

Steps to Implement Change: The baseline assessment consisted of obtaining a one-time snapshot for each floor on a specific day. The admission, BPMH and AMR information for each patient were recorded using the Electronic Patient Record and EMITT. A summary of this data was presented to pharmacists at meetings to help refine their practice. Two additional assessments were conducted to measure the change in the number of BPMHs and AMRs completed. Evaluation: Baseline measurements revealed BPMH and AMR hospital completion rates of $53 \%$ and $44 \%$ respectively. The average inpatient length of stay before BPMH completion was found to be 3.15 days. BPMH completion rates were found to vary depending on source of admission. By increasing pharmacists' awareness of the different sources of admissions, BPMH and AMR completion rates increased to $74 \%$ and $56 \%$ after two weeks, and $83 \%$ and $63 \%$ after four weeks. The average inpatient length of stay before BPMH completion also decreased to 2.8 and 1.7 days after 2 and 4 weeks, respectively. A residual group of long stay (palliative, alternate level of care) patients without AMR completed consistently affected results in all assessments.

Importance and Usefulness to Current Practice: By using an electronic tool to facilitate data collection, the proportion of patients with a completed BPMH and AMR increased by developing strategies that focused on vulnerable entry points.

\section{Teaching Skills Rotation for Pharmacy Residents Joan Lee, Patty McEwen, Kim Botsford, Mary Thornewell, Christine Wynne, Marita Tonkin} Hamilton Health Sciences, Hamilton, Ontario

Rationale: There is demand and need for the acquirement of teaching skills in pharmacy residency programs. Currently, limited opportunities exist for attainment of teaching skills.

Description: A longitudinal teaching skills rotation was developed for pharmacy residents at an academic teaching hospital. It is comprised of three components: 1) Curriculum - Small group sessions expose residents to basic teaching theory and principles. Topics include: teaching portfolio, evidence-based teaching, andragogy, learning and teaching styles, questioning technique, feedback provision and conflict management. One session is dedicated to theory application through mock teaching. 2) Practicum - Residents are matched with undergraduate pharmacy students in clinical rotations and assigned teaching responsibilities under the supervision of a clinical pharmacist. Examples of teaching activities include: therapeutic discussion, medication counseling, documentation, review of patient care plan. 3) Evaluation - Teaching skills rotation preceptor, clinical pharmacists, students and residents complete written and verbal evaluations on residents' performance.

Development \& Implementation: Pharmacy administrators, clinical pharmacists and residents were consulted. A rotation was piloted from January to April 2007 using a preceptor-resident-student model. Formal evaluations were completed to assess the value of the rotation and its impact on pharmacist workload. Based on initial feedback, additions were made to the curriculum and an elective teaching skills rotation was implemented for the 2007-2008 residency year. Ongoing stakeholder evaluations were conducted for reassessment and improvement of the rotation. Currently, the rotation is a mandatory component of the institution's residency program.

Evaluation: Stakeholder feedback indicated that resident teaching contributed to student learning with minimal impact on the clinical pharmacists' workload. Residents felt that training was invaluable and strongly recommended similar training for future residents.

Conclusion: Skills and knowledge acquired from a teaching skills rotation are valuable for residents' future participation in preceptorship and academia. It is recommended that teaching skills be incorporated into residency curricula.

Encore Presentation 


\section{PPC 2009 POSTER ABSTRACTS / RÉSUMÉS DES AFFICHES DE LA CPP 2009}

\section{Medication Reconciliation for Elective Surgical Admissions}

\author{
Karen McFarlane, Patty Brown, Shellyna Moledina, Alice Hogg, \\ Georgina Mikhail \\ Markham Stouffville Hospital Corporation, Markham, Ontario
}

Medication reconciliation is a well recognized method of improving patient safety. The process for surgeons to order patients' usual medications postoperatively at our institution was inconsistent and resulted in significant discrepancies between usual therapy and medications in hospital.

Our concept was for a Doctors' Order sheet to be generated from the computerized medication history completed by the nurses on all elective surgical patients in the surgical assessment clinic (SAC). An electronic form was created which lists the medication history and contains "yes", "no" and "hold" boxes for the physician to check. On the day of surgery, nurses in Day Surgery update any medication changes since the SAC appointment in the electronic record, then the order sheet is printed and placed on the chart which the surgeon completes post-operatively.

To evaluate the accuracy of the medication histories recorded in SAC, a Pharmacy Department representative performed a review of 17 patients on the surgical ward between Jan. and Feb. 2007. A variety of sources were used to compile a best possible medication history (BPMH) including patient/caregiver recall, medication vials, community pharmacy, family physician and provincial drug plan history. This BPMH was compared with the SAC history and variances were identified. The results showed a mean of 0.294 undocumented intentional discrepancies and 0.649 unintentional discrepancies per patient. This resulted in a medication reconciliation success index of $89.54 \%$.

The audit result demonstrates that order sheets obtained from SAC histories are an accurate and effective method of ensuring that patients' usual medications are ordered post-operatively.

Encore Presentation

\section{Examination of the Long-Acting Beta-Agonist Formulary Policy and Inhaler Technique}

\section{Jessica Stovel, Charles Bayliff, Santosh Deshpande} London Health Sciences Centre, London, Ontario

Rationale: London Health Sciences Centre (LHSC) implemented a long-acting beta-agonist (LABA) auto-substitution formulary policy which requires that patients ordered a combination LABA-inhaled corticosteroid (ICS) inhaler must use their own inhaler or receive a separate inhaler for each drug component.

Objectives: The primary objective was to determine the proportion of patients using their own inhaler. Secondary objectives included: comparing inhaler technique between those using their own and those subject to auto-substitution policy; comparing technique prior to and following an education intervention; and assessing amount of education received in the past.

Study Design and Methods: In this prospective, non-randomized, unblinded, observational study, 34 adult patients meeting criteria were interviewed. Inhaler technique before and after an education intervention was assessed according to an essential steps checklist. Patients were divided into two groups: Patient's Own Inhaler (PO) and Auto-substitution (AS). Inhaler technique scores were compared between groups prior to and after the pharmacist's educational intervention.

Results: Seventy-nine percent of patients were willing to use their own inhalers in hospital. Inhaler technique by patients subject to the LABA auto-substitution did not reveal a statistically significant difference when compared to that of those patients using their own inhaler: $4.5 / 7 \pm 1.2(\mathrm{PO})$ vs. $4.9 / 7 \pm 1.1$ (AS) $(P=0.32)$. We found that education resulted in a statistically significant improvement in inhaler technique: $4.9 / 7 \pm 1.1$ pre-education vs. $6.2 / 7 \pm 1.1$ post-education $(\mathrm{P}=0.0001)$. In addition, it was determined that $97 \%$ of patients received inhaler technique education with their initial prescription by the following health care professionals: physicians (47\%), pharmacists (37\%), and nurses (13\%). However, $79 \%$ of patients did not receive follow-up education throughout the course of their therapy.

Conclusions: Most patients are willing to use their own inhaler while in hospital. Those patients subject to the LABA auto-substitution formulary policy showed no statistically significant difference in inhaler technique. Education significantly improved short-term inhaler technique, was initially demonstrated to most patients, but rarely subsequently reviewed.

\section{Empiric Vancomycin Dosing Guidelines Based on Pharmacokinetics in Patients on Continuous Venovenous Hemodialysis}

L. van de Vijsel, S.A.N. Walker, S.E. Walker, S. Yamashita, A. Simor, $M$. Hladunewich

Sunnybrook Health Sciences Centre, Toronto, Ontario

Rationale: Evaluating the pharmacokinetics of vancomycin in ICU patients on continuous venovenous hemodialysis (CVVHD) will provide essential data in the investigation of empiric dosing guidelines.

Objectives: 1 . To determine the pharmacokinetics of vancomycin in ICU patients on CVVHD; 2. To propose and assess practical empiric dosing recommendations that target trough concentrations between $15-20 \mathrm{mg} / \mathrm{L} ; 3$. To identify and evaluate patient variables that significantly affect vancomycin pharmacokinetics.

Study Design and Methods: A retrospective chart review was conducted in 27 adult ICU patients. Empiric dosing recommendations were proposed using mean pharmacokinetic parameters and were evaluated using Monte Carlo simulation. Pearson's correlation analysis $(\mathrm{p}<0.2)$, followed by multiple linear regression $(\mathrm{p}<0.05)$ identified covariates that affect vancomycin pharmacokinetics. Significant covariates were modeled for the line of best fit and the regression equation was assessed using Pearson's correlation.

Results: The mean $( \pm$ SD) pharmacokinetic parameters determined for vancomycin were: $\mathrm{ke}$ of $0.033(0.02) ; \mathrm{t}_{1 / 2}$ of $28.2 \mathrm{~h}(16.8) ; \mathrm{Vd}$ of $1.2 \mathrm{~L} / \mathrm{kg}(0.7)$; and $\mathrm{AUC}_{24}$ of $266.8 \mathrm{mg} \cdot \mathrm{h} / \mathrm{L}$ (135.1). Vancomycin $1250 \mathrm{mg}$ IV q24h may be a reasonable empiric regimen, however, the probability of achieving desired trough concentrations was $12.2 \%$. The number of days on vancomycin correlated with ke $(\mathrm{p}=0.003)$, and a trend was observed with APACHE II score with $\mathrm{ke}(\mathrm{p}=0.053)$. The equation for the line of best fit was: $\mathrm{ke}=0.0631^{*}$ (\# days on vancomycin) $-0.4503\left(r^{2}=0.44 ; p=0.0003\right)$.

Conclusions: ICU patients managed with CVVHD demonstrated considerable intersubject variability. Although vancomycin $1250 \mathrm{mg}$ IV q24h may be a reasonable empiric regimen, follow-up therapeutic drug monitoring is essential. Our observations raise concerns about the reliability of numerous empiric dosing recommendations derived from small pharmacokinetic studies in heterogeneous populations.

Encore Presentation

\section{Cesium Induced Cardiac Arrest}

Matthew Wiens, ${ }^{1,2}$ Wendy Gordon, ${ }^{2,3}$ Daisy Baulcomb, ${ }^{4}$ Andre Mattman, 4,5 Tom Mock, ${ }^{4,5}$ Robert Brown ${ }^{3}$

'Chilliwack General Hospital, Chilliwack, British Columbia; ${ }^{2}$ Faculty of Pharmaceutical Sciences, University of British Columbia, Vancouver, British Columbia; ${ }^{3}$ Royal Columbian Hospital, New Westminster, British Columbia; ${ }^{4}$ Children's \& Women's Health Centre, Vancouver, British Columbia; ${ }^{5}$ Faculty of Medicine, University of British Columbia, Vancouver, British Columbia

Rationale: The chloride salt of cesium, a group 1A element, is gaining popularity as an alternative treatment of advanced cancers. To date several case reports have been published describing the arrythmogenic effects of cesium chloride, but none report on the pharmacokinetics.

Case Description: A 45 year old female was brought to the emergency department (ED) due to a syncopal episode. Her past medical history was significant for breast cancer. She had been treating herself with cesium chloride for the past 3 months. In the ED she experienced repeated episodes torsades de pointes requiring cardioversion. Serial cesium plasma and whole blood levels were measured over the ensuing 3 months and pharmacokinetic analysis was performed. Her ECG showed a prolonged QTc interval. The half life of cesium was determined to be 45 days. It was also noted that cesium sequesters in red blood cells.

Causality Assessment: There was a clear temporal relation between cesium ingestion and repeated episodes of tordades. No other explanation for repeated episodes of torsades de pointes could be found. All investigations were normal. The patient was not receiving any other drugs known to cause QT prolongation. It is well established that cesium chloride causes arrhythmia as it has been used in cardiovascular research for arrhythmogenesis in animals due to its potassium blocking effects.

Literature Evaluation: A rationale for the use of cesium in the treatment of cancer was described in 1984, by AK Brewer who hypothesized that cesium established alkaline conditions inside neoplastic cells, which ultimately resulted in apoptosis. Evidence of benefit in human cancer are limited brief case reports and one case series published in 1984 by HE Sartori. Clinicians must familiarize themselves with current trends in natural therapies and be prepared to discuss the dangers of these options with patients. 


\section{Development and Evaluation of a Multi-hospital Structured Pharmacy Orientation Program with an Emphasis on Patient Safety}

Donna MM Woloschuk,' Colette B Raymond ${ }^{2}$

'Regional Pharmacy Manager, Education; 'Staff Development I

Practice Evaluation Pharmacist, Winnipeg Regional Health Authority, Department of Pharmaceutical Services, Health Sciences Centre, Winnipeg, Manitoba

Rationale: Well-designed orientation programs are a key component of effective patient safety and risk management systems. Orientation programs may decrease adjustment time for new positions, improve job satisfaction and reduce turnover Literature describing orientation programs for pharmacy personnel is scarce and evaluation of those programs is rarely described.

Description of Concept: The Winnipeg Regional Health Authority Pharmacy program (nine hospital pharmacies) developed a new employee orientation program with an emphasis on patient safety, during a period of critical staffing shortfalls.

Steps Taken to Implement a New Program: We developed a comprehensive orientation program consisting of a detailed orientation checklist and 32 hours of interactive group orientation sessions (IGOS). Topics included hospital pharmacy overview, medication safety and problem prescriptions, drug information, communication, dispensary clinical practice, therapeutic drug monitoring, pharmacy care and the interdisciplinary team. Since inception in 2005, 55 new employees (85\% pharmacists) have participated in the IGOS.

Evaluation: Of 42 pharmacists who completed evaluations three months post-orientation, $83 \%$ indicated that they were satisfied or highly satisfied with training received. The IGOS that new pharmacists rated as most important and had subsequently applied in professional practice included: drug allergy assessment, high alert medication monitoring, efficient prescription triage, drug information skills and communication with other health professionals. New pharmacy employees emphasized the importance of supportive people for the transition to competent practitioners.

Importance to Practice: Participants valued the multi-hospital collaborative orientation program for new pharmacy employees. Sessions with patient safety implications were rated most important and immediately applicable to practice.

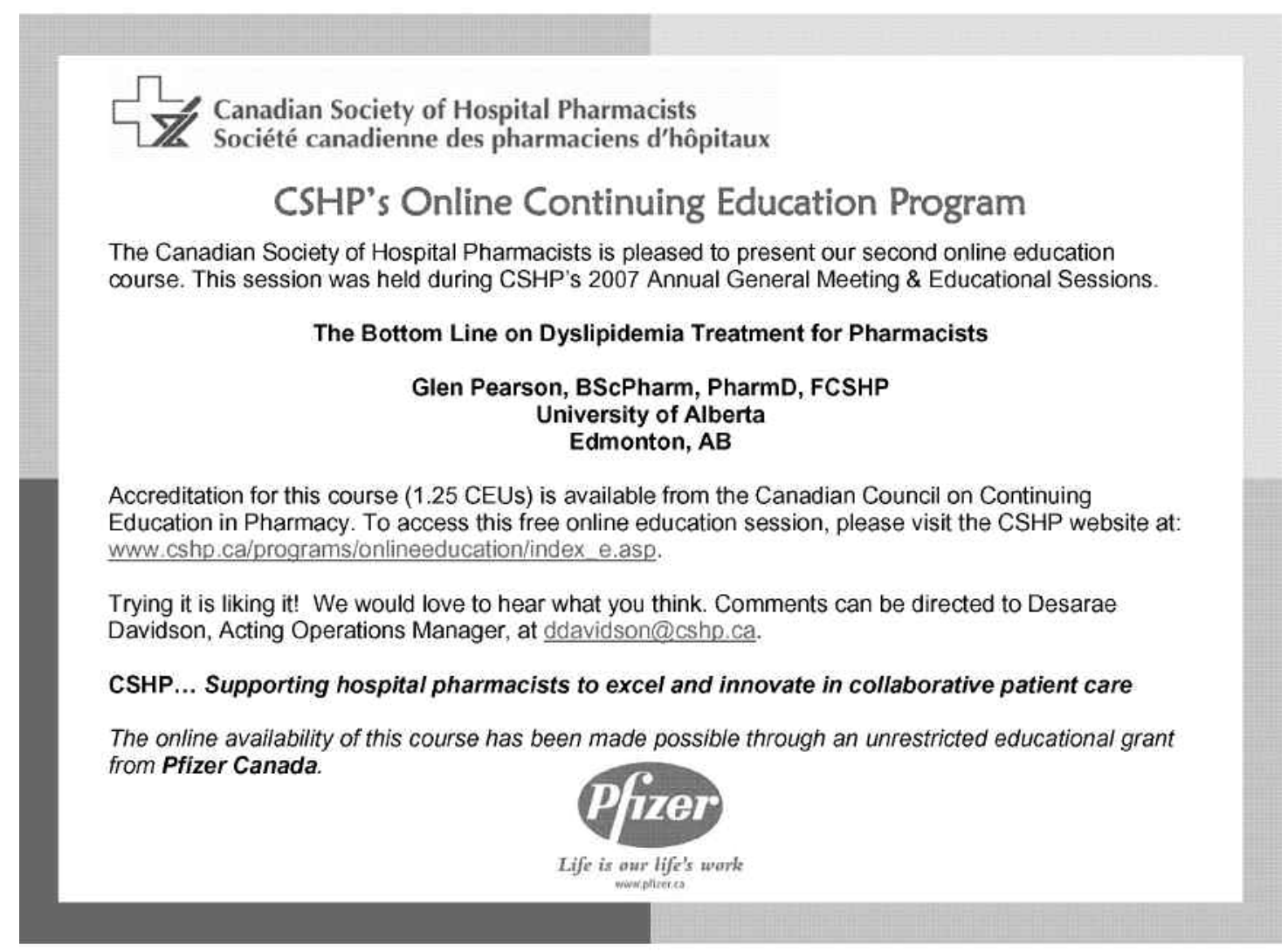

